\title{
iTRAQ-based Proteomic Analysis of a Chlorophyll- Deficient Mutant Caused by Single Base Change in RPS4 of Chinese Cabbage (Brassica Campestris L. ssp. Pekinensis)
}

\section{Xiaoyan Tang}

Anhui Agricultural University

\section{Fengyan Shi}

Shenyang Agricultural University

\section{Yiheng Wang}

Tianjin Academy of Agricultural Sciences

\section{Shengnan Huang}

Shenyang Agricultural University

\section{Ying Zhao}

Shenyang Agricultural University

Hui Feng ( $\nabla$ fenghuiaaa@syau.edu.cn )

Shenyang Agricultural University https://orcid.org/0000-0002-6345-6633

\section{Research article}

Keywords: Chinese cabbage, plastome mutant, iTRAQ, leaf proteome, RPS4

Posted Date: August 20th, 2020

DOI: https://doi.org/10.21203/rs.3.rs-56863/v1

License: (c) (1) This work is licensed under a Creative Commons Attribution 4.0 International License.

Read Full License 


\section{Abstract}

Background: Plastids are important plant-cell organelles containing a genome and bacterial-type $70 \mathrm{~S}$ ribosomes-primarily composed of plastid ribosomal proteins and ribosomal RNAs. In this study, a chlorophyll-deficient mutant $(\mathrm{cdm})$ obtained from double-haploid Chinese cabbage 'FT' was identified as a plastome mutant with an A-to-C base substitution in the plastid gene encoding the ribosomal protein RPS4. To further elucidate the function and regulatory mechanisms of RPS4, a comparative proteomic analysis was conducted between $c d m$ and ' $\mathrm{FT}$ ' plants using isobaric tags and a relative and absolute quantitation by (iTRAQ)-based strategy.

Results: A total of 6,245 proteins were identified, 540 of which were differentially expressed (DEPs) in the leaves of $c d m$ as compared to those of ' $F T^{\prime}$ '-including 233 upregulated and 307 downregulated proteins. Upregulated DEPs were mainly involved in translation, organic nitrogen synthesis, ribosomes, and spliceosomes. Meanwhile, downregulated DEPs were mainly involved in photosynthesis, photosynthetic reaction centres, photosynthetic light harvesting, carbon fixation, and chlorophyll binding. Our findings indicate an important role of RPS4 in the regulation of growth and development of Chinese cabbage, possibly by regulating plastid translation activity by affecting the expression of specific photosynthesisand cold stress-related proteins. Moreover, a multiple reaction monitoring test and quantitative real time polymerase chain reaction analysis confirmed our iTRAQ results.

Conclusions: Quantitative proteomic analysis allowed us to confirm diverse changes in the metabolic pathways between $c d m$ and 'FT' plants. Gene Ontology and the Kyoto Encyclopedia of Genes and Genomes enrichment analyses revealed that DEPs were significantly associated with photosynthesis, chlorophyll metabolism, carbon metabolism, RNA transport, glucosinolate biosynthesis, and gene splicing. This work provides new insights into the regulation of chlorophyll biosynthesis and photosynthesis in Chinese cabbage.

\section{Background}

Ribosomes are large ribonucleoprotein complexes composed of ribosomal proteins intertwined with rRNAs and are the site of cellular protein synthesis [1,2]. Ribosomes convert the genetic information in mRNAs into amino acids; thus, synthesising the encoded protein. Protein synthesis in plants occurs in the cytoplasm, plastids, and mitochondria, which contain their own unique ribosomal complex components [1]. Ribosomes can be classified into two categories according to their sedimentation coefficient-80S ribosomes are found in the cytoplasm of eukaryotic cells, and the $70 \mathrm{~S}$ ribosomes are found in prokaryotes and chloroplasts and mitochondria of higher plants. The chloroplast ribosome is composed of two subunits, large (50S) and small (30S). The $30 \mathrm{~S}$ subunit is composed of 24 ribosomal proteins and a $16 \mathrm{~S}$ rRNA, including 12 nuclear genes and 12 chloroplast genes. In turn, the $50 \mathrm{~S}$ subunit is composed of 33 ribosomal proteins and 3 rRNAs $-4.5 S, 5 S$, and 23S [3]. Twenty-four of the 33 ribosomal proteins are encoded by nuclear genes and the rest by plastid genes. Ribosomes read the genetic information contained in the nucleotide sequence of an mRNA and convert it into the sequence information of the 
corresponding amino acids to synthesise the encoded protein. Protein synthesis in plants occurs mainly in the cytoplasm, plastids and mitochondria, which contain their own unique ribosome complex components [1]. Ribosomal proteins are encoded by what are considered as the housekeeping genes. Regulation of ribosomal gene expression is important in prokaryotes and eukaryotes [4]. For example, there are 227 genes in the Arabidopsis genome encoding for its 80 ribosomal proteins-including 48 genes for the large subunit and 32 for the small subunit [5].

Some plastid ribosomal proteins (PRPs) are encoded by nuclear genes, whereas others are encoded by plastid genes [6]. In contrast, all chloroplast rRNA genes (16S, 23S, 5S, and $4.5 \mathrm{~s}$ ) are found in the plastid genome. PRPs are classified as essential or non-essential. Mutations in PRPs can severely damage plant photosynthesis and development, and the proteins involved are called essential PRPs. In contrast, mutated PRPs that have no significant impact on the translation or accumulation of plant ribosomes are called non-essential PRPs [7]. Numerous studies on the effects of plastid ribosome mutations on plant growth and development have been reported. In tobacco, homozygous double knockout mutants $r p / 36$, rps 18 are reportedly lethal, and heterozygous $r p / 36, r p s 18$ plants show similar phenotypes with thin and curled leaves [8]. Similarly, knockout of ribosomal protein S18 in tobacco plants results in abnormal leaf development, suggesting that chloroplast-based translation plays an important role in plant development [9]. Furthermore, maize mutant $r p s 17$ has yellow leaves and decreased photosynthetic activity [10]. Additionally, Arabidopsis thaliana ghs1 (also known as rps21)-a sucrose-sensitive mutant-may be responsible for leaf dysplasia and impairment of chloroplast development [11]. PRPS1, PRPS17, and PRPL24 play important roles in plant photosynthesis and growth $[7,12]$. The prps 5 mutant phenotype was first identified in a large-scale reverse genetic screen, and it was found that PRPS5 is involved in embryonic development, but its specific function and mechanism of regulation of plant development remain unclear [13].

Plant growth and development comprise biochemical and physiological changes including sugar synthesis, plant hormone signalling, secondary metabolite biosynthesis, stress response, carbohydrate catabolism, and accumulation of antioxidant substances [14]. Differential proteomic analysis methods can identify the extent of change in protein abundance to allow identification of proteins responsible for the regulation of metabolic pathways in the process of plant growth and development [15]. Twodimensional polyacrylamide gel electrophoresis $(2 D E)$ is a traditional method used in proteomic analysis. However, low-abundance proteins are difficult to detect by this method [16]. In recent years, the use of isobaric tags for relative and absolute quantitation (iTRAQ) has become the preferred method, as they are efficient in overcoming the deficiencies of 2DE for a precise comparison of protein levels among different materials [17]. Proteomic analysis is widely used to reveal differential expression of proteins in plant growth and development under specific conditions [18]. To date, iTRAQ has been applied to study chloroplast development associated with photosynthesis in many plant species, including tea [19], soybean [20], and cucumber [21]. However, proteomic analysis of chlorophyll-deficient mutants of Chinese cabbage has not yet been reported. 
Multiple reaction monitoring (MRM) is a powerful protein-targeting quantitative tool based on known or assumed reactive ion information. MRM specifically selects data for mass spectrometry signal collection, records ions meeting the rules, removes the interference of non-conforming ion signals, and obtains quantitative mass spectrometry information through the statistical analysis of the data [22]. Based on information quantisation signals, MRM can provide accurate and reproducible data for evaluating peptide and protein abundance; thus, improving the efficiency of protein expression verification [23].

Chlorophyll plays an important role in light harvesting and energy transfer in photosynthesis [24]. Furthermore, chlorophyll metabolism is associated with chloroplast development, photomorphogenesis, and plastid and nuclear signal transduction [25]. Although chlorophyll biosynthesis is relatively simple, its regulation, including that of its degradation, their effects on photosynthesis, and how they play a role in the light system remain unknown, thus warranting further study. Chlorophyll-deficient mutants are ideal materials for the exploration of the mechanisms of photosynthesis and chlorophyll synthesis [26, 27]; furthermore, many related genes have been identified [28-30]. However, the molecular mechanism underlying the effects of chlorophyll deficiency in chlorophyll-deficient mutants of Chinese cabbage remains unclear.

Chlorophyll-deficient mutant $c d m$ was isolated from an ethyl methanesulfonate (EMS) screen. $c d m$ plants exhibit pale-yellow inner leaves and slow growth. We identified a missense mutation in the plastid ribosomal protein S4 (RPS4) as responsible for the mutant phenotypes [31]. Here, we used an iTRAQbased quantitative proteomic approach to identify differentially expressed proteins (DEPs) in cdm as compared to wild-type 'Fukuda $50^{\prime}$ ' ('FT') Chinese cabbage leaves to identify the function and regulatory mechanism of candidate rps 4 gene associated with chlorophyll deficiency. Our results lay the foundation for the exploration of genetic and molecular mechanisms of plastid proteins in Chinese cabbage.

\section{Results}

\section{Characterisation of a maternally inherited chlorophyll deficiency mutation}

The chlorophyll-deficient mutant $(c d m)$ used herein was screened by EMS treatment of isolated microspores and proved to be a case of maternal inheritance [31]. All $c d m$ plants exhibited pale-yellow leaves, reduced growth, and severely altered chloroplast ultrastructure (Fig. 1). Compared with the wildtype ' $F T$ ', $c d m$ leaves had significantly lower net rates of photosynthesis $\left(\mathrm{P}_{\mathrm{n}}\right)$, stomatal conductance $\left(\mathrm{g}_{\mathrm{s}}\right)$, and transpiration $(\mathrm{E})$ and intercellular $\mathrm{CO}_{2}$ concentrations $\left(\mathrm{C}_{\mathrm{i}}\right)($ Table 1$)$. Our results indicate that the $c d m$ affects chlorophyll synthesis and chloroplast development, thereby affecting photosynthetic efficiency. 
Table 1

Photosynthetic characteristics of ' $\mathrm{FT}$ ' and $c d m$ at the seedling stage

\begin{tabular}{|c|c|c|c|c|}
\hline Variey & $\begin{array}{l}\text { Net photosynthetic } \\
\text { rate }\left(P_{n}\right) \\
\left(\mu \mathrm{mol} \cdot \mathrm{m}^{-2} \mathrm{~s}^{-1}\right)\end{array}$ & $\begin{array}{l}\text { Stomatal } \\
\text { conductance }\left(g_{s}\right) \\
\left(\mu \mathrm{mol} \cdot \mathrm{m}^{-2} \mathrm{~s}^{-1}\right)\end{array}$ & $\begin{array}{l}\text { Intercellular CO2 } \\
\text { concentration }\left(C_{i}\right) \\
\left(\mu \mathrm{mol} \cdot \mathrm{m}^{-2} \mathrm{~s}^{-1}\right)\end{array}$ & $\begin{array}{l}\text { Transpiration } \\
\text { rate }(E) \\
\left(\mu \mathrm{mol} \cdot \mathrm{m}^{-2} \mathrm{~s}^{-1}\right)\end{array}$ \\
\hline $\begin{array}{l}18.52 \pm \\
0.45^{\star}\end{array}$ & $0.21 \pm 0.01 *$ & $5.59 \pm 0.05^{\star}$ & $378 \pm 7.89 *$ & \\
\hline$c d m$ & $11.21 \pm 0.03$ & $0.13 \pm 0.45$ & $3.01 \pm 0.41$ & $311 \pm 5.83$ \\
\hline
\end{tabular}

\section{Quantitative Identification And Analysis Of Leaf Proteins}

To investigate DEPs associated with the maternally inherited $c d m$, proteomic analysis of $c d m$ and ' $F T^{\prime}$ ' was performed. Six samples-including three independent replicates, $c d m-1, c d m-2, c d m-3, \mathrm{FT}-1, \mathrm{FT}-2$, and FT-3-were labelled by iTRAQ tag, and 672,463 spectra were generated, 120,475 of which matched known peptides. The distribution of peptide-sequence coverage and charge statistics are shown in Figure S1. Protein coverage was highest at $0-5 \%$, followed by $5-10 \%$, and lowest at $35-40 \%$. Peptide length concentrated at 7-17 amino acid residues, with most identifiable proteins containing more than 11 peptides. In terms of mass and PI distribution, good coverage (0-2.3\% of total proteins in each molecular group) was obtained for a wide range of molecular weights (9-289 kDa), while PI varied from 3.8-12.2. A total of 6,245 proteins were identified in the Brassica_rapa_20100830.fasta library (Additional file 2: Table S2).

We next annotated 5,396 proteins using gene ontology (GO) analysis, 3,417 of which were annotated as 'cellular component', distributed between 'cell constituent' (90.4\%), 'cytoplasm' (73.5\%), 'organelle' (65.5\%), 'chloroplast' (23.4\%), 'nucleus' (15\%), 'chloroplast stroma' (9.1\%), 'cell membrane' (40.4\%), 'thylakoid' (7.8\%), and 'photosystem' (1.8\%). Additionally, 4,036 proteins were annotated as 'biological processes', significantly enriched for 'cell process' (73.5\%), 'metabolic process' (72\%), 'single-organism process' (46.6\%), 'cellular biosynthetic process' (32.8\%), 'response to stimuli' (18.8\%), 'gene expression' (18.3\%), 'translation' (13.2\%), and 'photosynthesis' (3.7\%). Lastly, 4,737 proteins were annotated as 'molecular function', significantly enriched for 'catalytic activity' (66.9\%), 'binding' (60.4\%), 'hydrolase activity' (22.7\%), 'transferase activity' (20.9\%), 'oxidoreductase activity' (14.2\%), 'structural molecule activity' (10.0\%), 'transporter activity' (6.3\%), 'peptidase activity' (4.9\%), and 'chlorophyll binding' (0.3\%) (Additional file 3: Table S3).

In addition, a Kyoto Encyclopedia of Genes and Genomes (KEGG) metabolic pathway analysis revealed 3,896 proteins enriched in 129 pathways including, 'metabolic pathway' (42.1\%), 'secondary metabolite biosynthesis' (24.8\%), 'ribosome synthesis' (10.6\%), 'carbon metabolism' (8.3\%), 'amino acid biosynthesis' 
(6.5\%), 'protein processing in endoplasmic reticulum' (5.8\%), 'endocytosis' (5.3\%), 'RNA transport' (5.2\%), 'spliceosome' (4.1\%), 'plant-pathogen interaction' (4.1\%), 'oxidative phosphorylation' (3.9\%), 'amino sugar and nucleotide sugar metabolism' (3.5\%), 'carbon fixation in photosynthetic organisms' (3.0\%), 'ribosome biogenesis in eukaryotes' (2.7\%), 'acetaldehyde glyoxylate and dicarboxylate metabolism' (2.6\%), 'glycine, serine, and threonine metabolism' (2.4\%), 'glycolysis/gluconeogenesis' (2.4\%), 'photosynthesis' (2.1\%), 'fructose and mannose metabolism' (1.9\%), 'pentose phosphate pathway' (1.8\%), 'peroxisome' (1.7\%), 'glyceride metabolism' ('glycerolipid metabolism', 1.0\%), 'photosynthesis-antenna protein' (0.8\%), among other metabolic pathways (Additional file 4: Table S4).

\section{Differences in leaf protein composition in cdm as compared to ' $\mathrm{FT}$ ' leaves maternally inherited chlorophyll-deficient mutation}

Comparative analysis of protein expression in $c d m$ and ' $F T$ ' leaves revealed 540 DEPs with an expression difference greater than 1.2-fold ( $p<0.05)$. In $c d m$ (relative to 'FT') $233(43.1 \%)$ DEPs were upregulated and 307 (56.9\%) downregulated. Furthermore, 170 DEPs were detected in cdm using MRM, of which 71 were upregulated and 75 downregulated. Further, MRM data agreed with iTRAQ data (Fig. 2, Additional file 5: Table S5).

DEPs were classified by GO analysis into three groups-cellular components, biological processes, and molecular functions. $\mathrm{GO}$ enrichment analysis annotated 310 proteins in different cellular components, including 'cell part' (90.3\%), 'cytoplasm' (73.5\%), 'organelle' (71.9\%), 'cell membrane' (45.5\%), 'chloroplast' (36.8\%), 'nucleus' (12.9\%), 'chloroplast stroma' (10.0\%), 'thylakoid' (19.4\%), and 'photosystem' (9.7\%). Based on biological process properties, 337 proteins were classified into 'cellular process' (81.0\%), 'metabolic process' (80.1\%), 'single-organism process' (40.0\%), 'cellular biosynthetic process' (31.2\%), 'gene expression' (22.6\%), 'translation' (17.5\%), and 'photosynthesis' (17.2\%). Furthermore, 400 DEPs were categorised in the 'molecular function' category, and were enriched for 'catalytic activity' (53.5\%), 'binding' (65.6\%), 'hydrolase activity' (17.8\%), 'transferase activity' (13.3\%), 'oxidoreductase activity' (14.0\%), 'structural molecule activity' (13.8\%), 'transporter activity' (8.0\%), 'peptidase activity' (2.8\%), and 'chlorophyll binding' (2.8\%) (Additional file 6: Table S6).

The 540 DEPs were further analysed using KEGG pathway, allowing us to determine that 333 of these proteins were enriched in 94 metabolic pathways. Significantly enriched pathways were sorted according to the number of proteins, including 'metabolic pathway' (48.0\%), 'biosynthesis of secondary metabolites' (20.1\%), 'ribosome synthesis' (17.1\%), 'carbon metabolism' (13.5\%), 'photosynthesis' (10.5\%), 'protein processing in endoplasmic reticulum' (7.2\%), 'glyoxylate and dicarboxylate metabolism' (6.6\%), 'carbon fixation in photosynthetic organisms' (6.3\%), 'photosynthesis-antenna proteins' (6.0\%), 'RNA transport' (5.1\%), 'amino acid biosynthesis' (4.2\%), 'glycine, serine, and threonine metabolism' (3.6\%), 'amino sugar and nucleotide sugar metabolism' (3.6\%), 'glycolysis/gluconeogenesis' (3.3\%), 'pentose phosphate pathway' (3.0\%), 'peroxisome metabolism' (3.0\%), 'fructose and mannose metabolism' (2.7\%), 'oxidative phosphorylation' (2.7\%), 'ribosome biogenesis in eukaryotes' (2.7\%), and 'glycerolipid metabolism' (2.4\%) 
(Additional file 7: Table S7). Comparative analysis of DEPs between cdm and 'FT' improves our understanding of chlorophyll deficiency mechanisms in Chinese cabbage.

\section{Characterisation of downregulated expression proteins (DRPs) in cdm and their role in the cdm phenotype}

Enrichment analyses between $c d m$ and 'FT' allowed for the annotation of 255 and 194 DRPs using GO and KEGG pathway analyses, respectively (Additional file 8: Table S8, Additional file 9: Table S9). The 255 GO DRPs were significantly enriched in the 'cell-component' categories, including 'thylakoid' $(p=3.49 \mathrm{e}-$ 58), 'photosynthetic membrane' ( $p=4.73 \mathrm{e}-55)$, 'chloroplast' $(p=1.44 \mathrm{e}-53)$, 'photosystem' $(p=1.33 \mathrm{e}-42)$, 'photosystem $\otimes^{\prime}(p=1.57 \mathrm{e}-19)$, and 'photosystem $\otimes^{\prime}(p=3.32 \mathrm{e}-26)$. In terms of 'biological processes', DRPs were strongly enriched in 'photosynthesis' $(p=2.67 \mathrm{e}-75)$, 'photosynthetic reaction centre' $(p=2.41 \mathrm{e}-40)$, 'generation of precursor metabolites' ( $p=8.15 \mathrm{e}-34)$, 'photosynthetic light harvesting' ( $p=4.41 \mathrm{e}-29)$, 'metabolic process' ( $p=3.30 \mathrm{e}-27)$, 'protein chromophore linkage' ( $p=1.63 \mathrm{e}-12)$, 'photorespiration' ( $p=$ 3.77e-12), 'carbon fixation' ( $p=3.27 \mathrm{e}-11)$. In 'molecular functions', DRPs were significantly enriched in 'chlorophyll binding' ( $p=2.37 \mathrm{e}-19)$, 'catalytic activity' $(p=3.01 \mathrm{e}-11)$, 'tetrapyrrole binding' $(p=1.23 \mathrm{e}-10)$, and 'oxidoreductase activity' ( $p=1.76 \mathrm{e}-09)$ (Fig. 3a).

The 194 DRPs identified by KEGG pathway analysis were strongly enriched in 'photosynthesis' $(p=5.04 \mathrm{e}-$ 47), 'metabolic pathways' ( $p=7.51 \mathrm{e}-40)$, 'photosynthesis-antenna proteins' $(p=9.06 \mathrm{e}-33)$, 'carbon metabolism' ( $p=5.82 \mathrm{e}-26)$, 'glyoxylate and dicarboxylate metabolism' ( $p=4.21 \mathrm{e}-19)$, 'carbon fixation in photosynthetic organisms' ( $p=5.88 \mathrm{e}-17)$, 'biosynthesis of secondary metabolites' ( $p=4.22 \mathrm{e}-07)$, 'pentose phosphate pathway' ( $p=4.19 \mathrm{e}-06)$, 'glycolysis/gluconeogenesis' $(p=6.53 \mathrm{e}-06)$, and 'glycine, serine, and threonine metabolism' ( $p=6.85 \mathrm{e}-06)$ (Fig. 3c).

Compared to 'FT' plants, photosynthesis is impaired in $c d m$ [31]. Consistently, 58 (29.9\%) of the DRPs were enriched in the biological process of photosynthesis. Most of these proteins were enriched in the same metabolic pathway, including 'photosystem \' (PSBB, PSBC, PSBE, PSBO, PSBP-1, PSBQ, and PSBR) and 'photosystem $\otimes^{\prime}$ in chloroplast proteins, as well as PETA and PETC, which were involved in light and electron transport. Expression of 17 light-harvesting proteins (LHCA1, LHCA2, LHCA3, LHCA4, LHCA6, LHCB1, CAB1, CAB2, LHCB1B1, LHCB1B2, LHCB2.1, LHCB2.3, LHCB3, LHCB4, LHCB4.2, LHCB5, and LHCB6) and 14 proteins associated with carbon cycle metabolism was significantly reduced (Table 2). The DRPs associated with impaired photosynthetic capacity may be related to the chlorophyll deficiency of $c d m$ leaves. Moreover, we observed that mutants exhibited a more obvious yellowing and slow growth in the greenhouse during winter. Further, a low-temperature treatment was performed at $4{ }^{\circ} \mathrm{C}$. After 24-h cold treatment, it was found that $c d m$ plants became more yellow than those grown at $26^{\circ} \mathrm{C}$ (Fig. 4). In contrast, no differences were observed between ' $\mathrm{FT}$ ' plants grown at $4{ }^{\circ} \mathrm{C}$ and $26^{\circ} \mathrm{C}$. Consistent with these findings, we identified a class of DRPs annotated as 'cold-stress response' (1.42e-04) (Table 3). 
Table 2

Identified DRPs associated with chlorophyll deficiency

\section{Annotation}

Gene-ID
Homologous in A. thaliana
ITRAQ- MRMratio

ratio

Photosynthesis

Photosystem II $47 \mathrm{kDa}$ protein (PSBB)

Bra040977 ATCG00680 0.68

0.50

photosystem II reaction center protein C (PSBC)

Bra041123 ATCG00280 0.53

photosystem II reaction center protein E (PSBE)

Bra041107

ATCG00580

0.48

0.26

Photosystem II manganese-stabilizing protein (PSBO)

$\begin{array}{llll}\text { Bra037164 AT5G66570 } & 0.64 & 0.64\end{array}$

photosystem II subunit P-1 (PSBP-1)

Bra015520 AT1G06680 0.78

photosystem II subunit Q-2 (PSBQ)

Bra029563 AT4G05180

0.70

0.50

photosystem II subunit R (PSBR)

Bra008392

AT1G79040

0.64

Photosystem I P700 chlorophyll a apoprotein A1 (PSAA)

Bra041122

ATCG00350

0.58

0.35

photosystem I subunit D-1 (PSAD-1)

Bra036240

AT4G02770

0.73

0.57

photosystem I subunit E-2 (PSAE-2)

Bra010350

AT2G20260

0.74

0.50

photosystem I subunit F (PSAF)

Bra038418

AT1G31330

0.61

photosystem I subunit G (PSAG)

Bra030843

AT1G55670

0.46

photosystem I subunit H2 (PSAH2)

Bra014317

AT1G52230

0.62

photosystem I subunit K (PSAK)

Bra010774

AT1G30380

0.51

photosystem I subunit L (PSAL)

Bra032672 AT4G12800

0.50

photosystem I reaction center subunit PSI-N,

chloroplast, putative / PSI-N, putative (PSAN)

$\begin{array}{lll}\text { Bra037761 AT5G64040 } & 0.60\end{array}$

photosynthetic electron transfer A (PETA)

Bra041106

ATCG00540

0.60

photosynthetic electron transfer C (PETC)

Bra000837

AT4G03280

0.61

0.30

photosynthetic electron transfer C (PETC)

Bra034200

AT4G03280

0.67

0.52

thylakoid lumenal $29.8 \mathrm{kDa}$ protein

Bra015696

AT1G77090

0.81

ferredoxin-NADP (+)-oxidoreductase 2 (FNR2)

Bra012203

AT1G20020

0.83

oxygen evolving enhancer 3 (PsbQ) family protein (PQL1)

Bra019675 AT1G14150

0.49

Chlorophyll A-B binding family protein (NPQ4)

Bra014024 AT1G44575 0.66 
Antenna proteins (light-harvesting chlorophyll protein complex)

$\begin{array}{llll}\text { Light-harvesting complex I chlorophyll a/b binding } & \text { Bra003198 } & \text { AT3G54890 } & 0.63\end{array}$ protein 1 (LHCA1)

$\begin{array}{llll}\text { Light-harvesting complex I chlorophyll a/b binding } \quad \text { Bra003451 AT3G61470 } & 0.72\end{array}$ protein2 (LHCA2)

$\begin{array}{llll}\text { Light-harvesting complex I chlorophyll a/b binding } & \text { Bra031427 } & \text { AT1G61520 } & 0.80\end{array}$ protein 3 (LHCA3)

Light-harvesting complex I chlorophyll a/b binding protein 4 (LHCA4)

Light-harvesting complex I chlorophyll a/b binding protein 6 (LHCA6)

Light-harvesting complex II chlorophyll a/b binding protein 1 (LhCB1)

Light-harvesting complex II chlorophyll a/b binding protein 1.1 (CAB2)

Light-harvesting complex II chlorophyll a/b binding protein 1.3 (CAB1)

Light-harvesting complex II chlorophyll a/b binding protein 1.4 (LHCB1B1)

Light-harvesting complex II chlorophyll a/b binding protein 1.5 (LHCB1B2)

Light-harvesting complex II chlorophyll a/b binding protein 2.1 (LHCB2.1)

Light-harvesting complex II chlorophyll a/b binding protein 2.3 (LHCB2.3)

Light-harvesting complex II chlorophyll a/b binding protein 3 (LHCB3)

Light-harvesting complex II chlorophyll a/b binding protein 4 (LHCB4)

Light-harvesting complex II chlorophyll a/b binding Bra029732 protein 4.2 (LHCB4.2)

Light-harvesting complex II chlorophyll a/b binding protein5 (LHCB5)

Light-harvesting complex II chlorophyll a/b binding protein6 (LHCB6)

Carbon fixation in photosynthetic organisms $\begin{array}{llll}\text { Bra018144 AT3G47470 } & 0.74 & 0.68\end{array}$

Bra016522 AT1G19150 0.83

Bra005425 AT2G34430 $\quad 0.50$

Bra010807 AT1G29920 0.72

Bra030182 AT1G29930

0.70

0.65

Bra005425

AT2G34430

0.50

Bra021909

AT2G34420

0.74

Bra013183

AT2G05100

0.68

Bra039070

AT3G27690

0.69

Bra002999

AT5G54270

0.63

0.81

Bra004989

AT2G40100

0.65

0.64

AT3G08940

0.70

0.53

Bra037913 AT4G10340

0.75

0.78

Bra026099

AT1G15820

0.57 


\begin{tabular}{|c|c|c|c|c|}
\hline Annotation & Gene-ID & $\begin{array}{l}\text { Homologous } \\
\text { in A. thaliana }\end{array}$ & $\begin{array}{l}\text { iTRAQ- } \\
\text { ratio }\end{array}$ & $\begin{array}{l}\text { MRM } \\
\text { ratio }\end{array}$ \\
\hline Ribulose-bisphosphate carboxylases (RBCL) & Bra028087 & ATCG00490 & 0.55 & 0.29 \\
\hline phosphoglycerate kinase 1 (PGK1) & Bra001470 & AT3G12780 & 0.81 & 0.56 \\
\hline Aldolase superfamily protein (ASP) & Bra026426 & AT4G26530 & 0.78 & 0.42 \\
\hline Fructose-bisphosphate aldolase (FBA) & Bra010717 & AT4G38970 & 0.83 & 0.73 \\
\hline $\begin{array}{l}\text { Peroxisomal NAD-malate dehydrogenase } \\
\text { (PMDH2) }\end{array}$ & Bra009397 & AT5G09660 & 0.75 & 0.79 \\
\hline $\begin{array}{l}\text { Alanine-2-oxoglutarate aminotransferase } 2 \\
\text { (AOAT2) }\end{array}$ & Bra016202 & AT1G70580 & 0.82 & \\
\hline Phosphoribulokinase (PRK) & Bra023235 & AT1G32060 & 0.78 & \\
\hline $\begin{array}{l}\text { Glyceraldehyde-3-phosphate dehydrogenase B } \\
\text { subunit (GAPB) }\end{array}$ & Bra034927 & AT1G42970 & 0.83 & 0.53 \\
\hline high cyclic electron flow 1 (HCEF1) & Bra007041 & AT3G54050 & 0.82 & 0.83 \\
\hline $\begin{array}{l}\text { glyceraldehyde 3-phosphate dehydrogenase A } \\
\text { subunit (GAPA) }\end{array}$ & Bra025219 & AT3G26650 & 0.83 & \\
\hline $\begin{array}{l}\text { glyceraldehyde 3-phosphate dehydrogenase A } \\
\text { subunit } 2 \text { (GAPA2) }\end{array}$ & Bra026948 & AT1G12900 & 0.74 & 0.48 \\
\hline Fructose-1,6-bisphosphatase (FBP) & Bra014005 & AT1G43670 & 0.79 & 0.48 \\
\hline $\begin{array}{l}\text { ribulose bisphosphate carboxylase small chain 3B } \\
\text { (AST3B) }\end{array}$ & Bra025431 & AT5G38410 & 0.51 & 0.32 \\
\hline Sedoheptulose-bisphosphatase (SBPASE) & Bra014720 & AT3G55800 & 0.81 & \\
\hline
\end{tabular}


Table 3

Identified DRPs in response to cold stress

\begin{tabular}{|c|c|c|c|c|}
\hline Annotation & Gene-ID & $\begin{array}{l}\text { Homologous } \\
\text { in A. } \\
\text { thaliana }\end{array}$ & $\begin{array}{l}\text { ITRAQ- } \\
\text { ratio }\end{array}$ & $\begin{array}{l}\text { MRM- } \\
\text { ratio }\end{array}$ \\
\hline catalase 2 (CAT2) & Bra017693 & AT4G35090 & 0.62 & 0.39 \\
\hline phosphoglycerate kinase 1 (PGK1) & Bra001470 & AT3G12780 & 0.81 & 0.56 \\
\hline $\begin{array}{l}\text { glyceraldehyde-3-phosphate dehydrogenase B } \\
\text { subunit (GABP) }\end{array}$ & Bra034927 & AT1G42970 & 0.83 & 0.53 \\
\hline Phosphoglucomutase (PGM) & Bra028278 & AT5G51820 & 0.79 & 0.79 \\
\hline Fibrillin (FIB) & Bra029481 & AT4G04020 & 0.66 & 0.65 \\
\hline chloroplast beta-amylase (CT-BMY) & Bra012676 & AT4G17090 & 0.75 & \\
\hline $\begin{array}{l}\text { Cold regulated } 314 \text { thylakoid membrane } 2 \\
\text { (COR314-TM2) }\end{array}$ & Bra032315 & AT1G29390 & 0.73 & \\
\hline \multicolumn{5}{|c|}{ Characterisation of upregulated expression proteins (URPs) in cdm } \\
\hline \multicolumn{5}{|c|}{$\begin{array}{l}\text { Enrichment analysis of URPs revealed } 194 \text { and } 139 \text { proteins annotated for metabolic pathways using } \\
\text { GO terms and KEGG pathway, respectively (Additional file } 10: \text { Table S10, Additional file } 11 \text { : Table } \\
\text { S11). The } 194 \text { URPs were significantly enriched in 'translation' }(p=4.27 \mathrm{e}-37) \text {, 'organic nitrogen } \\
\text { synthesis' }(p=7.15 \mathrm{e}-33) \text {, 'organic nitrogen metabolism' }(p=8.92 \mathrm{e}-30) \text {, 'cellular protein metabolism' ( } p \\
=9.79 \mathrm{e}-23) \text {, 'gene expression' }(p=2.4 \mathrm{e}-17) \text {, and 'protein folding' }(p=1.94 \mathrm{e}-16) \text { (Fig. 3b). }\end{array}$} \\
\hline \multicolumn{5}{|c|}{$\begin{array}{l}\text { In turn, KEGG pathway analysis of the } 139 \text { URPs showed that these proteins were enriched in seven } \\
\text { metabolic pathways including, 'ribosome assembling' }(p=3.79 \mathrm{e}-38) \text {, 'protein processing in } \\
\text { endoplasmic reticulum' }(p=2.99 \mathrm{e}-04) \text {, 'ribosome biogenesis in eukaryotes' }(p=3.15 \mathrm{e}-03) \text {, } \\
\text { 'spliceosome' }(p=3.51 \mathrm{e}-03) \text {, 'carbon metabolism' ( }(p=6.91 \mathrm{e}-03) \text {, 'RNA transport' }(p=0.033) \text {, and } \\
\text { 'glucosinolate biosynthesis' ( }(p=0.038) \text { (Fig. 3d). Of these, } 4 \text { were related to methylation, } 2 \text { to } \\
\text { transcription and translation termination, } 11 \text { to gene/protein splicing, and } 7 \text { to RNA degradation. } \\
\text { These data provide useful information for studying the molecular mechanisms underlying chlorophyll } \\
\text { deficiency in Chinese cabbage (Table 4). }\end{array}$} \\
\hline
\end{tabular}


Table 4

Identified URPs associated with chlorophyll deficiency

\begin{tabular}{|c|c|c|c|c|}
\hline Annotation & Gene-ID & $\begin{array}{l}\text { Homologous in } \\
\text { A. thaliana }\end{array}$ & $\begin{array}{l}\text { iTRAQ- } \\
\text { ratio }\end{array}$ & $\begin{array}{l}\text { MRM- } \\
\text { ratio }\end{array}$ \\
\hline \multicolumn{5}{|l|}{ Methylation } \\
\hline Fibrillarin 2 (FIB2) & Bra013905 & AT4G25630 & 1.28 & \\
\hline Methyltransferases & Bra025336 & AT3G28460 & 1.64 & \\
\hline Fibrillarin 2 (FIB2) & Bra010455 & AT4G25630 & 2.38 & \\
\hline Sun family protein (NOL1) & Bra039376 & AT3G13180 & 2.30 & \\
\hline \multicolumn{5}{|l|}{ Translational, translation termination } \\
\hline Mediator complex, subunit Med10 & Bra012447 & AT1G26665 & 1.28 & \\
\hline Rho termination factor & Bra022830 & AT1G06190 & 2.19 & \\
\hline \multicolumn{5}{|l|}{ Spliceosome } \\
\hline Mitochondrial HSO70-2 (MTHSC70-2) & Bra006027 & AT5G09590 & 1.28 & \\
\hline UBP1-associated protein 2A (UBA2A) & Bra007277 & AT3G56860.5 & 1.32 & \\
\hline RNA-binding family protein (RRM) & Bra009581 & AT5G02530.2 & 1.36 & \\
\hline $\begin{array}{l}\text { Mitochondrial heat shock protein } 70-1 \\
\text { (MTHSC70-1) }\end{array}$ & Bra010620 & AT4G37910 & 1.23 & 1.68 \\
\hline Small nuclear ribonucleoprotein family protein & Bra015768 & AT1G20580 & 1.39 & 1.96 \\
\hline $\begin{array}{l}\text { Chloroplast heat shock protein } 70-1 \\
\text { (CPHSC70-1) }\end{array}$ & Bra019231 & AT4G24280 & 1.20 & \\
\hline Heat shock cognate protein $70-1$ (HSC70-1) & Bra018725 & AT5G02500 & 1.22 & \\
\hline RNA-binding family protein (RRM) & Bra020273 & AT5G59950 & 1.25 & \\
\hline $\begin{array}{l}\text { Proline-rich spliceosome-associated family } \\
\text { protein (PSP) }\end{array}$ & Bra020904 & AT4G21660 & 1.27 & \\
\hline Mitochondrial HSO70-2 (MTHSC70-2) & Bra028628 & AT5G09590 & 1.24 & \\
\hline $\begin{array}{l}\text { P-loop containing nucleoside triphosphate } \\
\text { hydrolases superfamily protein }\end{array}$ & Bra030869 & AT2G28600 & 1.44 & \\
\hline \multicolumn{5}{|l|}{ RNA degradation } \\
\hline Cpn60 chaperonin family protein (TCP-1) & Bra001507 & AT3G13470 & 1.27 & \\
\hline Chaperonin 60 beta (CPN60B) & Bra011919 & AT1G55490.2 & 1.37 & \\
\hline Chaperonin-60alpha (CPN60A) & Bra011985 & AT2G28000 & 1.36 & 1.22 \\
\hline
\end{tabular}




\begin{tabular}{|lllll|}
\hline Annotation & Gene-ID & $\begin{array}{l}\text { Homologous in } \\
\text { A. thaliana }\end{array}$ & $\begin{array}{l}\text { iTRAQ- } \\
\text { ratio }\end{array}$ & $\begin{array}{l}\text { MRM- } \\
\text { ratio }\end{array}$ \\
\hline DEA(D/H)-box RNA helicase family protein & Bra018747 & AT1G48650 & 1.47 & \\
\hline RNA-binding family protein (ATRBP45C) & Bra019078 & AT4G27000 & 1.21 & 1.85 \\
\hline Cpn60 chaperonin family protein (TCP-1) & Bra028922 & AT5G56500 & 1.22 & 2.25 \\
\hline Chaperonin 60 beta (CPN60B) & Bra030858 & AT1G55490.2 & 1.44 & \\
\hline
\end{tabular}

\section{Differential Abundance Analysis Of Proteins Encoded By Chloroplast Genes}

Previously, we identified a missense mutation in RPS4 that affected one of the proteins found in the small subunit of the chloroplast ribosome. Proteins directly bound to the small subunit of the chloroplast ribosome are responsible for the synthesis of proteins encoded by chloroplast genes [31]. We found that 14 of the 6,245 identified proteins were encoded by chloroplast genes, of which, 12 were DRPs (Table 5). 
Table 5

Identification of proteins encoded by chloroplast genes in Brassica rapa

\begin{tabular}{|lllll|}
\hline Annotation & Gene-ID & $\begin{array}{l}\text { Homologous in A. } \\
\text { thaliana }\end{array}$ & $\begin{array}{l}\text { iTRAQ- } \\
\text { ratio }\end{array}$ & $\begin{array}{c}\text { MRM- } \\
\text { ratio }\end{array}$ \\
\hline $\begin{array}{l}\text { Ribulose-bisphosphate carboxylases } \\
\text { (RBCL) }\end{array}$ & Bra028087 & ATCG00490 & 0.55 & 0.29 \\
\hline $\begin{array}{l}\text { Photosystem II reaction center protein B } \\
\text { (PSBB) }\end{array}$ & Bra040977 & ATCG00680 & 0.68 & 0.50 \\
\hline $\begin{array}{l}\text { Ribosomal protein L14 (RPL14) } \\
\text { Chloroplast ribosomal protein S3 (RPS3) }\end{array}$ & Bra040981 & ATCG00800 & 0.74 & 0.60 \\
\hline $\begin{array}{l}\text { Ribosomal protein L2 (RPL2) } \\
\text { Phosphoglucomutase (PETA) }\end{array}$ & Bra040982 & ATCG01310 & 0.65 & \\
\hline $\begin{array}{l}\text { photosystem II reaction center protein E } \\
\text { (PSBE) }\end{array}$ & Bra041107 & ATCG00580 & 0.81 & 0.89 \\
\hline $\begin{array}{l}\text { Ribulose-bisphosphate carboxylases } \\
\text { (RBCL) }\end{array}$ & Bra041116 & ATCG00490 & 0.48 & 0.26 \\
\hline ATP synthase subunit alpha (ATPA) & Bra041120 & ATCG00120 & 0.29 & \\
\hline $\begin{array}{l}\text { Photosystem I, PsaA/PsaB protein } \\
\text { (PSAA) }\end{array}$ & Bra041122 & ATCG00350 & 0.60 & 0.47 \\
\hline $\begin{array}{l}\text { Photosystem II reaction center protein C } \\
\text { (PSBC) }\end{array}$ & Bra041123 & ATCG00280 & 0.53 & 0.35 \\
\hline $\begin{array}{l}\text { Ribosomal protein S2 (RPS2) } \\
\text { RNA polymerase subunit alpha (RPOA) }\end{array}$ & Bra041038 & ATCG00160 & 0.68 & \\
\hline \begin{tabular}{l} 
Unfolded protein bindin (YCF4) \\
\hline
\end{tabular} & Bra041105 & ATCG00520 & 0.95 & \\
\hline
\end{tabular}

Information on proteins encoded by chloroplast genes in Brassica is limited. However, the chloroplast genome of Chinese cabbage is very similar to that of $A$. thaliana. Indeed, RPS4 orthologues in $A$. thaliana and Chinese cabbage share $96.5 \%$ amino acid sequence identity (Additional file 13: Figure S2). Protein data files in MGF format were used to map to the Arabidopsis database (TAIR 10-pep-20101214), and 2,346 proteins were identified, of which, 47 were encoded by chloroplast genes. Overall, 29 proteins had differential expression greater than 1.2 -fold $(p<0.05)$. Of these, 26 were downregulated and 3 were upregulated in $c d m$. MRM was performed again, and 23 proteins were detected from these DEPs, 21 of which were downregulated and 2 upregulated (Table 6). The MRM test and ITRAQ data are in agreement. 
Table 6

Identification of chloroplast gene encoded differential proteins in Arabidopsis

\begin{tabular}{|c|c|c|c|c|}
\hline Gene-ID & Annotation & P-Value & $\begin{array}{l}\text { ITRAQ- } \\
\text { ratio }\end{array}$ & $\begin{array}{l}\text { MRM- } \\
\text { ratio }\end{array}$ \\
\hline \multicolumn{5}{|l|}{ DRPs } \\
\hline ATCG00020 & photosystem II reaction center protein A (PSBA) & 0 & 0.62 & 0.49 \\
\hline ATCG00120 & ATP synthase subunit alpha (ATPA) & 0 & 0.60 & 0.44 \\
\hline ATCG00130 & $\begin{array}{l}\text { ATPase, F0 complex, subunit B/B', } \\
\text { bacterial/chloroplast (ATPF) }\end{array}$ & $\begin{array}{l}6.60 \mathrm{e}- \\
75\end{array}$ & 0.64 & 0.30 \\
\hline ATCG00140 & ATP synthase subunit $\mathrm{C}$ family protein (ATPH) & $\begin{array}{l}1.28 \mathrm{e}- \\
26\end{array}$ & 0.37 & \\
\hline ATCG00150 & ATPase, F0 complex, subunit A protein (ATPI) & $\begin{array}{l}8.42 \mathrm{e}- \\
23\end{array}$ & 0.59 & \\
\hline ATCG00160 & Ribosomal protein S2 (RPS2) & $\begin{array}{l}5.52 \mathrm{e}- \\
55\end{array}$ & 0.75 & 0.71 \\
\hline ATCG00270 & photosystem II reaction center protein D (PSBD) & $\begin{array}{l}1.4 \mathrm{e}- \\
252\end{array}$ & 0.61 & 0.43 \\
\hline ATCG00280 & photosystem II reaction center protein C (PSBC) & 0 & 0.64 & 0.42 \\
\hline ATCG00340 & Photosystem I, PsaA/PsaB protein (PSAB) & $\begin{array}{l}1.5 e- \\
210\end{array}$ & 0.60 & 0.40 \\
\hline ATCG00350 & Photosystem I, PsaA/PsaB protein (PSAA) & $\begin{array}{l}1.2 \mathrm{e}- \\
142\end{array}$ & 0.56 & 0.36 \\
\hline ATCG00380 & Chloroplast ribosomal protein S4 (RPS4) & $\begin{array}{l}1.3 e- \\
147\end{array}$ & 0.68 & 0.63 \\
\hline ATCG00420 & $\mathrm{NADH}$ dehydrogenase subunit $\mathrm{J}(\mathrm{NDHJ})$ & $\begin{array}{l}1.27 \mathrm{e}- \\
07\end{array}$ & 0.57 & \\
\hline ATCG00470 & ATP synthase epsilon chain (ATPE) & $\begin{array}{l}1.44 \mathrm{e}- \\
89\end{array}$ & 0.64 & 0.27 \\
\hline ATCG00480 & ATP synthase subunit beta (ATPB) & 0 & 0.64 & 0.50 \\
\hline ATCG00490 & Ribulose-bisphosphate carboxylases (RBCL) & 0 & 0.49 & 0.29 \\
\hline ATCG00540 & Photosynthetic electron transfer A (PETA) & $\begin{array}{l}5.8 \mathrm{e}- \\
233\end{array}$ & 0.60 & 0.40 \\
\hline ATCG00580 & Photosystem II reaction center protein E (PSBE) & $\begin{array}{l}4.07 e- \\
50\end{array}$ & 0.59 & 0.46 \\
\hline ATCG00600 & photosynthetic electron transport chain (PETG) & $\begin{array}{l}6.74 \mathrm{e}- \\
05\end{array}$ & 0.58 & \\
\hline
\end{tabular}




\begin{tabular}{|c|c|c|c|c|}
\hline Gene-ID & Annotation & P-Value & $\begin{array}{l}\text { iTRAQ- } \\
\text { ratio }\end{array}$ & $\begin{array}{l}\text { MRM- } \\
\text { ratio }\end{array}$ \\
\hline ATCG00680 & photosystem II reaction center protein B (PSBB) & 0 & 0.66 & 0.51 \\
\hline ATCG00710 & photosystem II reaction center protein $\mathrm{H}(\mathrm{PSBH})$ & $\begin{array}{l}3.39 \mathrm{e}- \\
38\end{array}$ & 0.55 & 0.45 \\
\hline ATCG00720 & photosynthetic electron transfer B (PETB) & $\begin{array}{l}1.37 e- \\
72\end{array}$ & 0.53 & 0.26 \\
\hline ATCG00730 & Photosynthetic electron transfer D (PETD) & $\begin{array}{l}5.03 e- \\
19\end{array}$ & 0.39 & 0.33 \\
\hline ATCG00770 & Ribosomal protein S8 (RPS8) & $\begin{array}{l}1.73 e- \\
42\end{array}$ & 0.74 & 0.77 \\
\hline ATCG00800 & Structural constituent of ribosome (RPS3) & $\begin{array}{l}4.12 \mathrm{e}- \\
94\end{array}$ & 0.71 & 0.69 \\
\hline ATCG01060 & $\begin{array}{l}\text { Photosynthetic electron transport in photosystem I } \\
\text { (PSAC) }\end{array}$ & $\begin{array}{l}1.82 \mathrm{e}- \\
101\end{array}$ & 0.62 & 0.40 \\
\hline ATCG01100 & NADH dehydrogenase family protein (NDHA) & $\begin{array}{l}1.25 \mathrm{e}- \\
10\end{array}$ & 0.46 & \\
\hline \multicolumn{5}{|l|}{ URPs } \\
\hline ATCG00180 & DNA-directed RNA polymerase family protein (RPOC1) & $\begin{array}{l}5.74 \mathrm{e}- \\
24\end{array}$ & 1.25 & 2.66 \\
\hline ATCG00650 & Ribosomal protein S18 (RPS18) & $\begin{array}{l}8.13 e- \\
06\end{array}$ & 1.36 & \\
\hline ATCG00740 & RNA polymerase subunit alpha (RPOA) & $\begin{array}{l}2.81 \mathrm{e}- \\
29\end{array}$ & 1.27 & 2.23 \\
\hline
\end{tabular}

\section{Transcriptional Expression Analysis Of Deps}

The expression patterns of some photosynthesis-related genes (Bra000837, Bra008392, Bra010350, Bra010774, Bra012203, Bra014024, Bra014317, Bra015520, Bra015696, Bra019675, Bra029563, Bra030843, Bra032672, Bra034200, Bra036240, Bra037164, Bra037761, and Bra038418) were analysed by quantitative real-time polymerase chain reaction (qRT-PCR) (Fig. 5). Expression levels of these genes were consistent with our findings from ITRAQ and MRM. The consistency between these various approaches demonstrates that the results obtained are reliable.

\section{Discussion}

Previously, we identified a Chinese cabbage mutant $c d m$ containing a missense mutation in the chloroplast gene rps4, resulting in a Gly to Val substitution at position 193 [31]; thus, enabling us to 
analyse the role of RPS4. RPS4 belongs to a class of proteins that are the first to bind to the 16S rRNA during prokaryotic ribosome assembly, and is an essential component of the $30 \mathrm{~S}$ ribosomal subunit [32]. In Escherichia coli, mutation of RPS4 affects the higher-order structure of the 16S rRNA, influencing the accuracy of translation [33]. We revealed that the maternally inherited mutation identified Chinese cabbage RPS 4 reduces the accumulation of chloroplast 23S, 16S, 5S, and 4.5 rRNAs, thereby impairing the processing of these rRNAs in $c d m$ and $c d m \times \triangle \mathrm{FT}^{\prime}$ plants [31]. Here, we showed that RPS 4 is likely associated with the processes of photosynthesis, plant development, and cold-stress resistance in Chinese cabbage.

Our quantitative proteomic analysis revealed that $c d m$ strongly affects the levels of many photosynthesis-related proteins, likely contributing to the mutant phenotypes of pale yellow leaves and slow overall growth. Proteomic analysis also suggested that RPS4 likely contributes to cold-stress resistance, supported by the performance of $c d m$ and 'FT' plants grown at 4 or $26^{\circ} \mathrm{C}$ (Fig. 4).

\section{Rps4 Is Required For Translation In The Plastid}

Defects to RPS4 can lead to aberrant rRNA processing [31], which likely affects ribosome function and translation in the plastid [8,34]. RPS4 is highly conserved in bacteria, yeast, higher plants, and animals. Further, it has been observed that mutations to yeast RPS4 influence the accuracy of the eukaryotic ribosome, suggesting that the crucial role of RPS4 in ribosome function is phylogenetically conserved [35]. After BLAST and homology-based searches against $A$. thaliana database, we found 14 proteins (of 6,245 proteins identified) encoded by plastid genes in leaves of $c d m$ and 'FT' plants. Of these 14 proteins, 12 were downregulated in $c d m$ leaves. The protein data files in MGF format were used to map to the $A$. thaliana database (TAIR 10-pep-20101214). A total of 2,346 proteins were identified, 47 of which are encoded by plastid genes -26 of which were downregulated and 3 upregulated in $c d m$ leaves. These results suggest that protein synthesis is impaired in the chloroplasts of $c d m$ leaves; thus, we suggest that the missense mutation in rps4 may lead to impaired plastid translation in $c d m$ Chinese cabbage.

\section{Global downregulation of specific protein groups causes chlorophyll deficiency in cdm}

To understand the effects of $c d m$ on protein synthesis, we performed comparative proteomic analysis and identified most of the DRPs as being involved in photosynthesis (Table 6). Some proteins, associated with the subunits of photosystems I and II, were downregulated in $c d m$ leaves. Consistently, photosynthetic capacity was also reduced in $c d m$ plants. GO analysis annotated 58 proteins $(29.9 \%)$ as involved in photosynthesis, including 9 proteins in photosystem I, 7 proteins in photosystem II, and 2 proteins in photosynthetic electron transport. Additionally, 17 proteins annotated as light-harvesting, and 14 involved in the carbon cycle metabolic pathway were significantly downregulated. The light-harvesting proteins of photosystems I and II strongly regulate light harvesting and are supposed to result in aberrant stacking of the grana in the chloroplast. In Arabidopsis, light-harvesting proteins are markedly reduced or entirely missing in chlorophyll-deficient mutants [36]; thus, light-harvesting proteins are assumed to be necessary for stacking of the thylakoid membranes. Carbon fixation is a crucial step in photosynthesis 
[37]. SBPase plays an important role in the Calvin cycle [38,39] and affects chloroplast biogenesis [40]. A slight reduction of SBPase activity might cause a significant decrease in the rate of $\mathrm{CO}_{2}$ fixation and light-saturated photosynthesis [41]. In our study, 14 proteins involved in carbon fixation were downregulated in $c d m$ leaves, which may partially explain the impairment of photosynthesis in these leaves. Furthermore, a reduction in the accumulation of some photosynthetic proteins (PSAA, PSAD, PSAF, PSBB, Lhca2, and RBCL) is reportedly responsible for defects in photosynthetic performance on account of severely reduced translational capacity $[7,42]$. The reduction of these essential photosynthetic protein components might explain the chlorophyll deficiency and slow growth observed in cdm plants. For reference, proteomic data (MGF search against the $A$. thaliana database) from iTRAQ and MRM analyses revealed that RPS4 was significantly decreased. Proteomic analysis suggests that RPS4 may affect a class present in both plastid and nuclear genomes.

We proposed that the substitution of glycine $(\mathrm{G})$ for valine $(\mathrm{V})$ at residue 193 in chloroplast RPS4 in $c d m$ plants leads to aberrant plastid rRNA processing, which in turn affects the synthesis of PRPs and downregulates some chloroplast-encoded genes involved in photosynthesis chloroplast development. The reduction in levels of these proteins leads to a reduction in proteins encoded by nuclear genes. Together, the downregulation of these two types of proteins results in the phenotype characteristics of cdm plants (Fig. 6).

\section{URPs may lead to the chlorophyll deficiency observed in cdm plants}

Many of the URPs in $c d m$ leaves are involved in post-transcriptional and post-translational modification. Consistently, URPs were considerably enriched in the 'spliceosome' KEGG pathway ( $p=3.51 \mathrm{e}-03)$. RNA splicing is a significant post-transcriptional event essential for the regulation of gene expression. Several pre-mRNA processing and splicing factors were upregulated in $c d m$ leaves, including the proline-rich spliceosome-associated family protein (PSP) -a splicing factor found in Arabidopsis [43]. UBP1associated protein 2A (UBA2A) is an RNA-binding protein (RBP) implicated in mRNA splicing and stability. Small nuclear ribonucleoprotein family protein $\left(\mathrm{SmD}_{3}\right)$ is crucial for the splicing of primary transcripts. $\mathrm{SmD}_{3}$ mutants exhibit delayed flowering and slow root growth; further, SmD3-b may be a major component of the spliceosomal small nuclear ribonucleoprotein in Arabidopsis [44]. RBPs have vital roles across all aspects of post-transcriptional gene regulation in eukaryotes. Transient expression of UBA2A in protoplasts might lead to increased enrichment of transporter mRNA [45]. Overall, post-transcriptional RNA and protein processing suggest essential steps in the regulation of gene expression in $c d m$ leaves and are likely associated with the global suppression of photosynthesis-related gene expression. Furthermore, methylation is implicated in gene-silencing, and four proteins involved in methylation were upregulated in $c d m$ leaves, including Fibrillarin 2-a key protein involved in rRNA methylation [46]. Our proteomics data suggest that proteins involved in methylation may influence the phenotypes of $\mathrm{cdm}$ plants.

Cold stress tolerance is reduced in cdm plants

Page $18 / 34$ 
We observed that $c d m, c d m \times$ ' $F T$ ', and $c d m \times$ WZ (a genetically-distant inbred line of Chinese cabbage) showed more obvious chlorophyll deficiency and slower growth when grown in a greenhouse during the winter than that when grown under high temperatures. A cold treatment experiment also suggests that $c d m$ plants are cold sensitive. Of 307 DRPs, GO analysis revealed that a class of proteins involved in cold-stress response was decreased in $c d m$ plants.

Previous studies have suggested that most of the PRPs are associated with plant development, but few studies have reported the involvement of PRPs in stress response. For example, Rp/33 plants did not show a distinct phenotype under standard growth conditions, but did when grown at $4{ }^{\circ} \mathrm{C}$, thus indicating that RPL33 plays a critical role in chilling stress response [47]. Similarly, a missense mutation in rps5 reduces plant cold tolerance and causes a severe reduction in chlorophyll content and seedling weight. Conversely, RPS5-overexpressing plants recovers from cold stress, suggesting an important function of RPS5 in cold-stress tolerance [42]. PRL4, RPL12, and a BPL21-homolog were also found to be involved in cold-stress response in Arabidopsis [48]. Downregulation of these cold stress-related proteins in cdm plants suggests a potential role for rps 4 in cold-stress response.

\section{Conclusions}

$c d m$, a Chinese cabbage line containing a maternally inherited chlorophyll-deficient mutation, exhibits impaired plastid translation and alteration to the expression of key components of photosystems I and II. Aberrant plastid rRNA processing and mutation of RPS4 seemingly affect the assembly of the chloroplast $30 S$ subunit, likely leading to the defective chlorophyll deficiency and slow growth phenotypes in $c d m$ plants. Furthermore, our study suggests a potential role for RPS4 in cold-stress tolerance.

\section{Methods}

\section{Plant materials}

The maternally inherited, chlorophyll-deficient mutant used in this study was obtained from a doublehaploid Chinese cabbage ' $\mathrm{FT}$ ' line after EMS mutagenic treatment of cultured isolated microspores [31]. In September 2016, 'FT' and $c d m$ seeds were germinated at $26^{\circ} \mathrm{C}$ for $24 \mathrm{~h}$ and sown in an aperture disk. Seedings were potted after 2 weeks and placed in the greenhouse at Shenyang Agricultural University in Shenyang, Liaoning Province, China. After 2 weeks, $c d m$ and ' $F T^{\prime}$ ' leaves were sampled, wrapped in aluminium foil, and frozen in liquid nitrogen. All samples for protein and RNA extraction were stored at $-80^{\circ} \mathrm{C}$. Three biological replicates of each genotype and treatment were prepared.

For cold treatment, 2-week old ' $\mathrm{FT}$ ' and $c d m$ seedings were placed in incubators (PERCIVAL, USA LT-36VL) set to different temperatures $\left(26^{\circ} \mathrm{C}\right.$ or $\left.4^{\circ} \mathrm{C}\right)$ for $24 \mathrm{~h}$.

\section{Photosynthetic Parameter Measurements}


Ten healthy, uniform, and vigorous plants each of $c d m$ and 'FT' were marked. The photosynthetic parameters of the selected plants were measured in the greenhouse using a CIRAS-2 portable gasexchange system (PP system company, USA). The net photosynthetic rate $\left(\mu \mathrm{mol} \cdot \mathrm{m}^{-2} \mathrm{~s}^{-1}\right)$, stomatal conductance $\left(\mu \mathrm{mol} \cdot \mathrm{m}^{-2} \mathrm{~s}^{-1}\right)$, intercellular $\mathrm{CO}_{2}$ concentration $\left(\mu \mathrm{mol} \cdot \mathrm{m}^{-2} \mathrm{~s}^{-1}\right)$, and transpiration rate $\left(\mu \mathrm{mol} \cdot \mathrm{m}^{-2} \mathrm{~s}^{-1}\right)$ of the third true leaf were measured at 9:00-11:00 on a sunny day. The light intensity was set to $1,200 \mu \mathrm{mol} \cdot \mathrm{m}^{-2} \mathrm{~s}^{-1}$, the leaf-chamber temperature to $30^{\circ} \mathrm{C}$, and the ambient $\mathrm{CO}_{2}$ concentration was used. Each plant was measured three times.

\section{Protein Extraction And Preparation}

For protein extraction, $\sim 1.5 \mathrm{~g}$ frozen leaf tissue was frozen in liquid nitrogen for grinding, after which 200 $\mu \mathrm{L}$ lysis buffer (8 M urea, $30 \mathrm{mM}$ HEPES, 1 mM PMSF, 2 mM EDTA, $10 \mathrm{mM}$ DTT) was added. Samples were then ultrasonicated for $5 \mathrm{~min}$ and centrifuged at $20,000 \times \mathrm{g}$ for $30 \mathrm{~min}$, after which the supernatant was collected. Subsequently, DTT was added to the samples to a final concentration of $10 \mathrm{mM}$ and incubated at $56^{\circ} \mathrm{C}$ for $1 \mathrm{~h}$. The samples were then drawn out and iodoacetamide (IAM) was added quickly, to a final concentration of $55 \mathrm{mM}$. Samples were then stored in the dark for $1 \mathrm{~h}$. Four volumes of pre-cooled acetone were then added to the samples, which were then kept at $-20^{\circ} \mathrm{C}$ for $3 \mathrm{~h}$ for precipitation, followed by centrifugation at $30,000 \times \mathrm{g}$ for $30 \mathrm{~min}$. The supernatant was discarded, and $300 \mu \mathrm{L}$ solution buffer ( $50 \%$ TEAB and $0.1 \%$ SDS) was added to the precipitate. Samples were ultrasonicated again for $3 \mathrm{~min}$ and centrifuged at 20,000 $\times \mathrm{g}$ for $30 \mathrm{~min}$. A Bradford assay was used to measure total protein concentration [49]. Protein quality was determined by $2 \mathrm{DE}$, in triplicate (Additional file 14: Figure. S3). Finally, protein samples were stored at $-80^{\circ} \mathrm{C}$ for further experiments. All centrifugations were performed at $4{ }^{\circ} \mathrm{C}$.

\section{Protein digestion, iTRAQ labelling, and high performance liquid chromatography (HPLC) (strong cation- exchange chromatography)}

Samples were processed in triplicate. For protein digestion, a volume containing $100 \mu \mathrm{g}$ of protein was added to each sample in $10 \mathrm{~K}$ ultrafiltration tubes and centrifuged at $14,000 \times \mathrm{g}$ for 40 min, after which, the liquid waste was discarded. Subsequently, $200 \mu \mathrm{L}$ of $50 \%$ TEAB was added to the samples and centrifuged again at $14,000 \times \mathrm{g}$ for $40 \mathrm{~min}$. Again, the liquid waste was discarded, and this step was repeated two times. Trypsin, $1 \mu \mathrm{g} \mu \mathrm{L}^{-1}$, was added along with $100 \mu \mathrm{g}$ of protein substrate to $3.3 \mu \mathrm{g}$ enzyme and incubated at $37^{\circ} \mathrm{C}$ for $24 \mathrm{~h}$. The digestion fluid was lyophilised, and $30 \mu \mathrm{L}$ TEAB $(1: 1$ water:TEAB) was added to solubilise the peptide segment. All centrifugations were performed at $4{ }^{\circ} \mathrm{C}$.

Peptide labelling was as follows: Tags 115,116 , and 117, for $c d m-1, c d m-2$, and $c d m-3$, respectively; Tags 118,119 , and 121 , for FT-1, FT-2, and FT-3, respectively. The labelling reagent was equilibrated at $25^{\circ} \mathrm{C}$. A volume of $70 \mu \mathrm{L}$ isopropanol was added to each labelling reagent, swirled for $1 \mathrm{~min}$, and centrifuged to the bottom of the tube. The mixed and labelled reagent was added to the peptide segment, and different 
samples were labelled with different sizes of isotopes. Samples were then kept at room temperature for $2 \mathrm{~h}$, after which, the labelled peptide mixtures were dried by vacuum centrifugation.

Strong cation-exchange chromatography was performed using an Agilent 1100 HPLC Pump System. The labelled samples were diluted 10 times with buffer $\mathrm{A}\left(25 \% \mathrm{ACN}\right.$ and $\left.10 \mathrm{mM} \mathrm{KH}_{2} \mathrm{PO}_{4}, \mathrm{PH} 3.0\right)$, and pH was adjusted to 3.0 with phosphoric acid. Samples were centrifuged at $15,000 \times \mathrm{g}$ for $10 \mathrm{~min}$, and the supernatant was collected. Samples were then eluted with buffer $B\left(25 \% \mathrm{ACN}, 2 \mathrm{M} \mathrm{KCL}, 10 \mathrm{mM} \mathrm{KH} \mathrm{PO}_{4}\right)$. After $35 \mathrm{~min}$, buffer B started to mix, and a peak appeared after $39 \mathrm{~min}$. A total of 30 fractions were collected over one-minute intervals, and these fractions were purified and desalted using a C18 reversedphase chromatography column (Phenomenex, Torrance, CA, USA).

\section{Liquid Chromatography-electrospray Ionisation-tandem Mass Spectrometry (ms/ms) Analysis}

Desalted peptide mixtures were added to an Acclaim PePmap C18-reversed phase column $(75 \mu \mathrm{m} \times 2 \mathrm{~cm}$, $3 \mu \mathrm{m}, 100 \AA$ a perture) (Eksigent, Silicon Valley, CA, USA) and separated in a reversed phase $\mathrm{C} 18$ column (75 $\mu \mathrm{m} \times 10 \mathrm{~cm}, 5 \mu \mathrm{m}, 300 \AA$ A aperture) (Eksigent) installed in a Dionex ultimate 3000 nano LC system. Peptides were eluted at a flow rate of $300 \mathrm{~nL} \mathrm{~min}^{-1}$ using $5-80 \%(\mathrm{v} / \mathrm{v})$ acetonitrile in $0.1 \%$ formic acid with a Q Exactive mass spectrometer (Thermo Fisher Scientific, Waltham, MA, USA).

Peptides were analysed by tandem MS/MS after nanoelectrospray ionisation in a Q Exactive mass spectrometer (Thermo Fisher Scientific). Intact peptides were detected at an orbital resolution of 70,000.

Using high-energy and collision-dissociation operation modes, 28.0 selected peptides were prepared for MS/MS analysis with standardised collision energy and detection of debris in orbital debris at a resolution of 17,500 . The process of alternately dependent data on an MS scan followed by applying the 20 most abundant precursor ions to the $20 \mathrm{MS} / \mathrm{MS}$ scans was performed following the Dynamic Exclusion duration of $15 \mathrm{~s}$. The applied electric spray voltage was $1.8 \mathrm{kV}$, and the automatic-gain control system was used to optimise the orbit generation spectrum. The automatic-gain control objectives of MS and MS2 were $3 \mathrm{e} 6$ and $1 \mathrm{e} 5$, respectively. The $\mathrm{m} / \mathrm{z}$ scan ranges of MS and MS2 were 350-2000 Da and > $100 \mathrm{Da}$, respectively.

\section{Analysis Of Protein Data And Identification Of Deps}

All protein data files obtained from the Orbitrap were converted to MGF format using Proteome Discoverer 1.2 (Thermo Fisher Scientific). Proteins were identified from the Brassica database (http://brassicadb.org/brad/index.php) using Mascot (Matrix Science, UK; version 2.3.02). Mass tolerance of the whole peptide mass and fragmented ions was set to a maximum of $0.05 \mathrm{Da}(\mathrm{ppm})$ and 0.1 Da, respectively. The potential variable modifications were Gln- > pyro-Glu (N-term Q), Oxidation (M), and Deamidated (NQ). The fixed modifications were Carbamidomethyl (C), iTRAQ8plex (N-term), and 
iTRAQ8plex (K). Charge states of peptides were +2 and +3 . In brief, by selecting the decoy checkbox, an automatic decoy database search was performed, which randomly generated the random database sequence and tested the original spectrum as well as the real database. To reduce the probability of false peptide recognition, only peptides with a confidence interval greater than 95\% (by Mascot probability analysis) were identified.

Identified peptides which contained at least two unique spectra were used for protein quantitation. The median ratio of Mascot was used to weigh and normalise quantitative protein ratios. Only the proteins that met the criteria of expression difference multiple $>1.2$-fold $(p<0.05)$ were considered differentially expressed.

\section{Bioinformatic Analysis And Functional Annotation}

GO is a method based on the known gene (protein) annotation to annotate the function of the gene (protein) under study. GO describes the biological processes, cellular components, and molecular functions of proteins [50]. In turn, the KEGG database is used to annotate the information identified at the biological pathway level, which is a set of path diagrams for molecular interactions and reaction networks [51].

Protein function was annotated using the Blast2GO programme against a non-redundant protein sequence database (http://www.geneontology.org/). Blastx/Blastp 2.2.24 + was used to map all identified DEPs to pathways using the KEGG database (http://www.genome.jp/kegg/genes.html). The significance of GO and KEGG pathway results was judged by the threshold value $p \leq 0.05$.

\section{Mrm Analysis}

MRM technology is used to validate the results of differential proteins, monitor target proteins, verify target protein information, and quantitatively compare target proteins from multiple samples.

Quantitative MRM analysis was conducted by monitoring the pre-selected transition signals of candidate proteins. Accordingly, establishing optimal transitions was key. An AB6600 mass spectrometer was used to detect peptide signals. ProteinPilot 5.0, Mascot 2.3.01, and Skyline 1.2.0.3425 were used to analyse MRM data.

\section{Qrt-pcr Analysis}

An RNAprep Pure Plant Kit (Tiangen, Beijing, China) was used to extract total RNA from $c d m$ and 'FT' leaves, following the manufacturer's instructions. An Agilent 2100 Bioanalyzer and $0.8 \%$ agarose gel electrophoresis were used to determine the quality and purity of extracted RNA, respectively. mRNA was purified from total RNA using oligo-dT magnetic beads, and cDNA was synthesised using SuperScript III, following the manufacturer's instructions. Diluted CDNA (1:10) was used for qRT-PCR, and primers 
(Additional file 1: Table S1) were designed using Primer 5.0. qRT-PCR was conducted on a Bio-Rad IQ5 as described previously [52]. The $2^{-\Delta \triangle C t}$ [53] formula was used to quantify relative gene expression levels. Each treatment was repeated three times, and data analysis was performed using the Bio-Rad IQ5 software.

\section{Abbreviations}

PRPs

Plastid ribosomal proteins; rRNAs:Ribosomal RNAs; MRM:Multiple reaction monitoring;

DEPs:Differentially expressed proteins; RPs:Ribosomal proteins; 2DE:Two-dimensional polyacrylamide gel electrophoresis; iTRAQ:Isobaric mass tagging; RPS4:Plastid ribosomal protein S4; GO:Gene Ontology; qRT-PCR:Quantitative real-time polymerase chain reaction; DRPs:Downregulated expression proteins; URPs:Upregulated expression proteins; PSP:Proline-rich spliceosome-associated family protein; UBA2A:UBP1-associated protein 2A; RBPs:RNA-binding proteins; $\mathrm{SmD}_{3}:$ Small nuclear ribonucleoprotein family protein

\section{Declarations}

\section{Ethics approval and consent to participate}

Not applicable.

\section{Consent for publication}

Not applicable.

\section{Availability of data and materials}

Mass spectrometry proteomics data have been deposited with the ProteomeXchange Consortium (http://proteomecentral.proteomexchange.org) via the iProX partner repository [54] with the dataset identifier PXD020683.

\section{Competing interests}

The authors declare that there are no competing interests.

\section{Funding}

This work was supported by a grant from the National Natural Science Foundation of China (No. 31730082).

\section{Author contributions}


$\mathrm{XYT}$ and FYS conducted experiments, performed data analysis, and wrote the manuscript. HF directed the study, including experimental design and manuscript revision. YHW, SNH and YZ participated in the creation of plant materials. All authors read and approved of the final manuscript.

\section{Acknowledgements}

We would like to thank the Shanghai luming biological technology co., LTD (Shanghai, China) for providing proteomics services.

\section{References}

1. Schippers JH, Mueller-Roeber B. Ribosomal composition and control of leaf development. Plant Sci. 2010;179:307-15.

2. Yusupov MM, Yusupova GZ, Baucom A, Lieberman K, Earnest TN, Cate J, et al. Crystal structure of the ribosome at 5.5 A resolution. Science. 2001;292(5518):883-96.

3. Yamaguchi K, Subramanian AR. Proteomic identification of all plastid-specific ribosomal proteins in higher plant chloroplast 30S ribosomal subunit. Eur J Biochem. 2003;270(2):190-205.

4. Lee KH, Park J, Williams DS, Xiong Y, Hwang I, Kang BH. Defective chloroplast development inhibits maintenance of normal levels of abscisic acid in a mutant of the Arabidopsis RH3 DEAD-box protein during early post-germination growth. Plant J. 2013;73(5):720-32.

5. Carroll AJ. The Arabidopsis Cytosolic Ribosomal Proteome: From form to Function. Front Plant Sci. 2013;4(1):32.

6. Fleischmann TT, Scharff LB, Alkatib S, Hasdorf S, Schottler MA, Bock R. Nonessential plastidencoded ribosomal proteins in tobacco: a developmental role for plastid translation and implications for reductive genome evolution. Plant Cell. 2011;23:3137-55.

7. Tiller N, Weingartner M, Thiele W, Maximova E, Schottler MA, Bock R. The plastid-specific ribosomal proteins of Arabidopsis thaliana can be divided into non-essential proteins and genuine ribosomal proteins. Plant J. 2012;69(2):302-16.

8. Tiller N, Bock R. The translational apparatus of plastids and its role in plant development. Mol Plant. 2014;7(7):1105-20.

9. Rogalski M, Ruf S, Bock R. Tobacco plastid ribosomal protein S18 is essential for cell survival. Nucleic Acids Res. 2006;34:4537-45.

10. Schultes NP, Sawers RJ, Brutnell TP, Krueger RW. Maize high chlorophyll fluorescent 60 mutation is caused by an Ac disruption of the gene encoding the chloroplast ribosomal small subunit protein 17 . Plant J. 2000;21:317-27.

11. Morita-Yamamuro C, Tsutsui T, Tanaka A, Yamaguchi J. Knock-out of the plastid ribosomal protein S21 causes impaired photosynthesis and sugar-response during germination and seedling development in Arabidopsis thaliana. Plant Cell Physiol. 2004;45(6):781-8. 
12. Romani I, Tadini L, Rossi F, Masiero S, Pribil M, Jahns P, et al. Versatile roles of Arabidopsis plastid ribosomal proteins in plant growth and development. Plant J. 2012;72:922-34.

13. Bryant N, Lloyd J, Sweeney C, Myouga F, Meinke D. Identification of nuclear genes encoding chloroplast-localized proteins required for embryo development in Arabidopsis. Plant Physiol. 2011;155(4):1678-89.

14. Giovannoni JJ. Genetic regulation of fruit development and ripening. Plant Cell. 2004;16, $170-80$.

15. Andrade JDM, Toledo TT, Nogueira SB, Cordenunsi BR, Lajolo FM. 2D-DIGE analysis of mango (Mangifera indica L.) fruit reveals major proteomic changes associated with ripening. J Proteomics. 2012;75(11):3331-41.

16. Zieske LR. A perspective on the use of ITRAQ reagent technology for protein complex and profiling studies. J Exp Bot. 2006;57(7):1501-8.

17. Evans C, Noirel J, Ow SY, Salim M, Pereira-Medrano AG, Couto N, et al. An insight into iTRAQ: where do we stand now? Anal Bioanal Chem. 2012;404(4):1011-27.

18. Fukao Y, Ferjani A, Tomioka R, Nagasaki N, Kurata R, Nishimori Y, et al. iTRAQ analysis reveals mechanisms of growth defects due to excess zinc in Arabidopsis. Plant Physiol. 2011;155(4):1893907.

19. Wang L, Cao H, Chen C, Yue C, Hao X, Yang Y, et al. Complementary transcriptomic and proteomic analyses of a chlorophyll-deficient tea plant cultivar reveal multiple metabolic pathway changes. $\mathrm{J}$ Proteomics. 2016;130:160-9.

20. Qin J, Zhang J, Liu D, Yin C, Wang F, Chen P, et al. iTRAQ-based analysis of developmental dynamics in the soybean leaf proteome reveals pathways associated with leaf photosynthetic rate. Mol Genet Genomics. 2016;291(4):1595-605.

21. Lei R, Du Z, Kong J, Li G, He Y, Qiu Y, Yan J, Zhu S. Blue Native/SDS-PAGE and iTRAQ-Based Chloroplasts Proteomics Analysis of Nicotiana tabacum Leaves Infected with M Strain of Cucumber Mosaic Virus Reveals Several Proteins Involved in Chlorosis Symptoms. Proteomics. 2018;18(2).

22. Addona TA, Abbatiello SE, Schilling B, Skates SJ, Mani DR, Bunk DM, et al. Multi-site assessment of the precision and reproducibility of multiple reaction monitoring-based measurements of proteins in plasma. Nat Biotechnol. 2009;27(7):633-41.

23. Abbatiello SE, Mani DR, Schilling B, Maclean B, Zimmerman LJ, Feng X, et al. Design, implementation and multisite evaluation of a system suitability protocol for the quantitative assessment of instrument performance in liquid chromatography-multiple reaction monitoring-MS (LC-MRM-MS). Mol Cell Proteomics. 2013;12(9):2623-39.

24. Liu W, Fu Y, Hu G, Si H, Zhu L, Wu C, et al. Identification and fine mapping of a thermo-sensitive chlorophyll deficient mutant in rice (Oryza sativa L.). Planta. 2007;226(3):785-95.

25. Eckhardt U, Grimm B, Hortensteiner S. Recent advances in chlorophyll biosynthesis and breakdown in higher plants. Plant Mol Biol. 2004;56(1):1-14.

26. Leon P, Arroyo A, Mackenzie S. Nuclear control of plastid and mitochondrial development in higher plants. Annu Rev Plant Physiol Plant Mol Biol. 1998;49(1):453-80. 
27. Liu YY, Wang X, Zhang CZ, Yang L, Gao DL, Chen HM, et al. A CsYcf54 variant conferring light green coloration in cucumber. Euphytica. 2016;208(3):509-17.

28. Li N, Jia J, Xia C, Liu X, Kong X. Characterization and mapping of novel chlorophyll deficient mutant genes in durum wheat. Breed Sci. 2013;63(2):169.

29. Sandhu D, Coleman Z, Atkinson T, Rai KM, Mendu V. Genetics and Physiology of the Nuclearly Inherited Yellow Foliar Mutants in Soybean. Front Plant Sci. 2018;9:471.

30. Sakowska K, Alberti G, Genesio L, Peressotti A, Delle Vedove G, Gianelle D, et al. Leaf and canopy photosynthesis of a chlorophyll deficient soybean mutant. Plant Cell Environ. 2018;41(6):1427-37.

31. Tang XY, Wang YH, Zhang Y, Huang SN, Liu ZY, Fei DL, et al. A missense mutation of plastid RPS4 is associated with chlorophyll deficiency in Chinese cabbage (Brassica campestris ssp. pekinensis). BMC Plant Biol. 2018;18(1):130.

32. Nowotny V, Nierhaus $\mathrm{KH}$. Assembly of the $30 \mathrm{~S}$ subunit from Escherichia coli ribosomes occurs via two assembly domains which are initiated by S4 and S7. Biochemistry. 1988;27:7051-5.

33. Allen PN, Noller HF. Mutations in ribosomal proteins S4 and S12 influence the higher order structure of 16S ribosomal RNA. J Molecular Biol. 1989;208, 457 - 68.

34. Wicke S, Schneeweiss GM, Müller KF, Quandt D. The evolution of the plastid chromosome in land plants: gene content, gene order, gene function. Plant Mol Biol. 2011;76(3-5):273-97.

35. Markus MA, Gerstner RB, Draper DE, Draper DE, Torchia DA. The solution structure of ribosomal protein $S 4 \Delta 41$ reveals two subdomains and a positively charged surface that may interact with RNA. EMBO J. 1988;17(16):4559-71.

36. Kim EH1, Li XP, Razeghifard R, Anderson JM, Niyogi KK, Pogson BJ, et al. The multiple roles of lightharvesting chlorophyll a/b-protein complexes define structure and optimize function of Arabidopsis chloroplasts: a study using two chlorophyll b-less mutants. Biochim Biophys Acta. 2009;1787(8):973-84.

37. Bar-Even A, Noor E, Lewis NE, Milo R. Design and Analysis of Synthetic Carbon Fixation Pathways. Proc Natl Acad Sci U S A. 2010;107:8889-94.

38. Lefebvre S, Lawson T, Zakhleniuk OV, Lloyd JC, Raines CA, Fryer M. Increased sedoheptulose-1,7bisphosphatase activity in transgenic tobacco plants stimulates photosynthesis and growth from an early stage in development. Plant Physiol. 2005;138(1):451-60.

39. Tamoi M, Nagaoka M, Miyagawa Y, Shigeoka S. Contribution of fructose-1,6-bisphosphatase and sedoheptulose-1,7-bisphosphatase to the photosynthetic rate and carbon flow in the Calvin cycle in transgenic plants. Plant Cell Physiol. 2006;47(3):380-90.

40. Liu XL, Yu HD, Guan Y, Li JK, Guo FQ. Carbonylation and loss-of-function analyses of SBPase reveal its metabolic interface role in oxidative stress, carbon assimilation, and multiple aspects of growth and development in Arabidopsis. Mol Plant. 2012;5(5):1082-99.

41. Olcer H, Lloyd JC, Raines CA. Photosynthetic capacity is differentially affected by reductions in sedoheptulose-1,7-bisphosphatase activity during leaf development in transgenic tobacco plants. Plant Physiol. 2001;125:982-9. 
42. Zhang JX, Yuan H, Yang Y, Fish T, Lyi SM, Thannhauser TW. Plastid ribosomal protein S5 is involved in photosynthesis, plant development, and cold stress tolerance in Arabidopsis. J Exp Bot. 2016;67:2731-44.

43. Wang BB, Brendel V. The ASRG database: identification and survey of Arabidopsis thaliana genes involved in pre-mRNA splicing. Genome Biol. 2004;5(12):R102.

44. Swaraz AM, Park YD, Hur Y. Knock-out mutations of Arabidopsis SmD3-b induce pleotropic phenotypes through altered transcript splicing. Plant Sci. 2011;180(5):661-71.

45. Lambermon MHL, Fu Y, Kirk DAW, Dupasquier M, Filipowicz W, Lorković ZJ. UBA1 and UBA2, two proteins that interact with UBP1, a multifunctional effector of pre-mRNA maturation in plants. Mol Cell Biol. 2002;22(12):4346-57.

46. Barneche F, Steinmetz F, Echeverria M. Fibrillarin genes encode both a conserved nucleolar protein and a novel small nucleolar RNA involved in ribosomal RNA methylation in Arabidopsis thaliana. J Biol Chem. 2000;275:27212-20.

47. Rogalski M, Schöttler MA, Thiele W, Schulze WX, Bock R. Rpl33, a nonessential plastid-encoded ribosomal protein in tobacco, is required under cold stress conditions. Plant Cell. 2008;20(8):222137.

48. Bae MS, Cho EJ, Choi EY, Park OK. Analysis of the Arabidopsis nuclear proteome and its response to cold stress. Plant J. 2003;36(5):652-63.

49. Bradford MM. A Rapid and Sensitive Method for the Quantitation of Microgram Quantities of Protein Utilizing the Principle of Protein-Dye Binding. Anal Biochem. 1976;72(1-2):248-54.

50. Yan $\mathrm{XH}$, Dong $\mathrm{CH}, \mathrm{Yu}$ JY, Liu WH, Jiang CH, Liu J, et al. Transcriptome profile analysis of young floral buds of fertile and sterile plants from the self-pollinated offspring of the hybrid between novel restorer line NR1 and Nsa CMS line in Brassica napus. BMC Genom. 2013;14(1):26.

51. Kanehisa M, Goto S, Sato Y, Furumichi M, Tanabe M. KEGG for integration and interpretation of largescale molecular data sets. Nucleic Acids Res. 2012;40:D109-14.

52. Shi FY, Zhou X, Liu ZY, Feng H. Rapid alkalinization factor (RALF) genes are related to genic male sterility in Chinese cabbage (Brassica rapa L. ssp. pekinensis). Scientia Horticulture. 2017;225:480-9.

53. Livak KJ, Schmittgen TD. Analysis of relative gene expression data using realtime quantitative PCR and the $2^{-\Delta \Delta C t}$ method. Methods. 2001;25(4):402-8.

54. Ma J, Chen T, Wu SF, Yang CY, Bai MZ, Shu KX, et al. iProX: an integrated proteome resource. Nucleic Acids Res. 2019;47(D1):D1211-7.

\section{Figures}



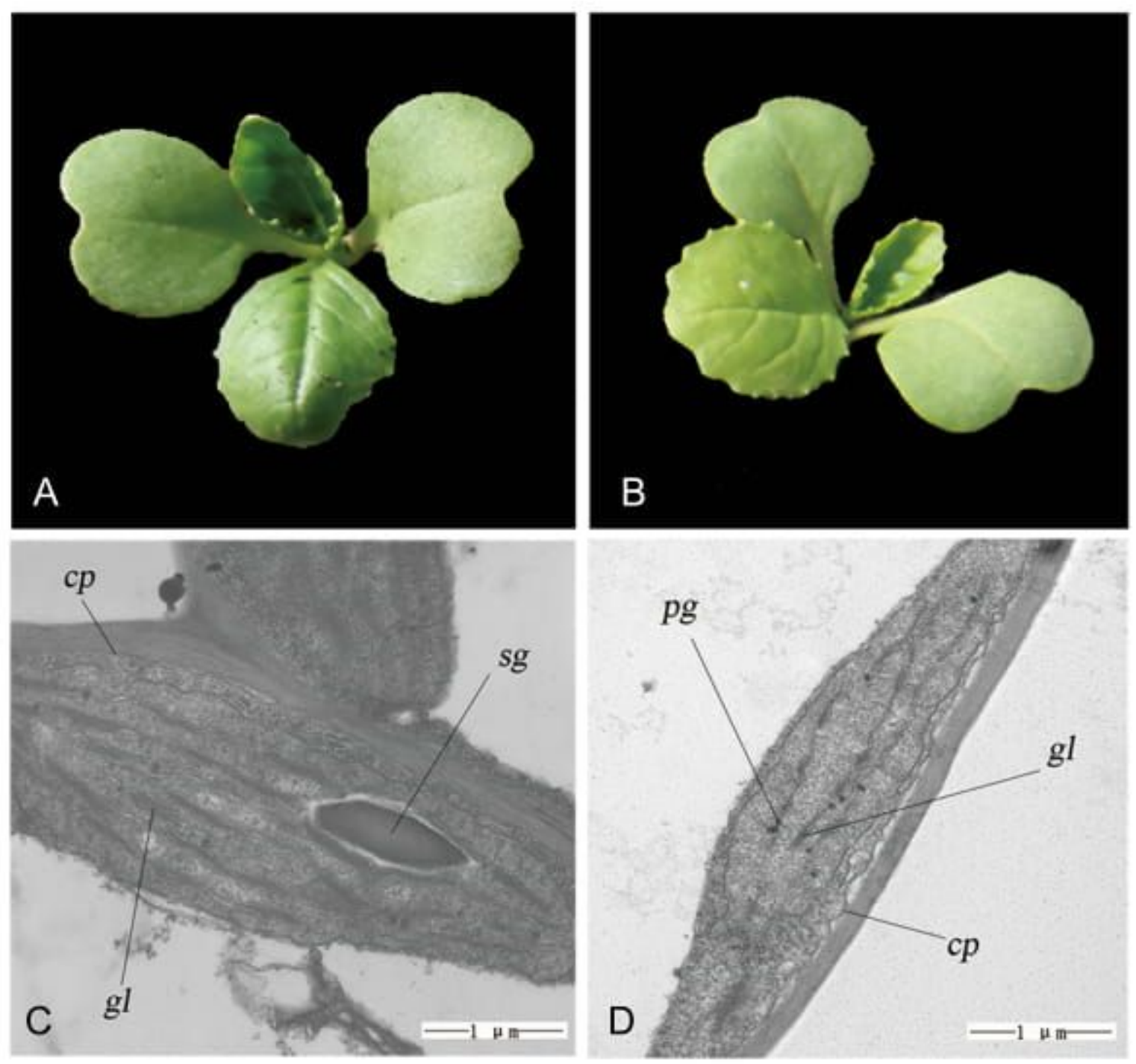

Figure 1

Plant and chloroplast ultrastructure of ' $\mathrm{FT}$ ' (a and c) and cdm (b and d) 


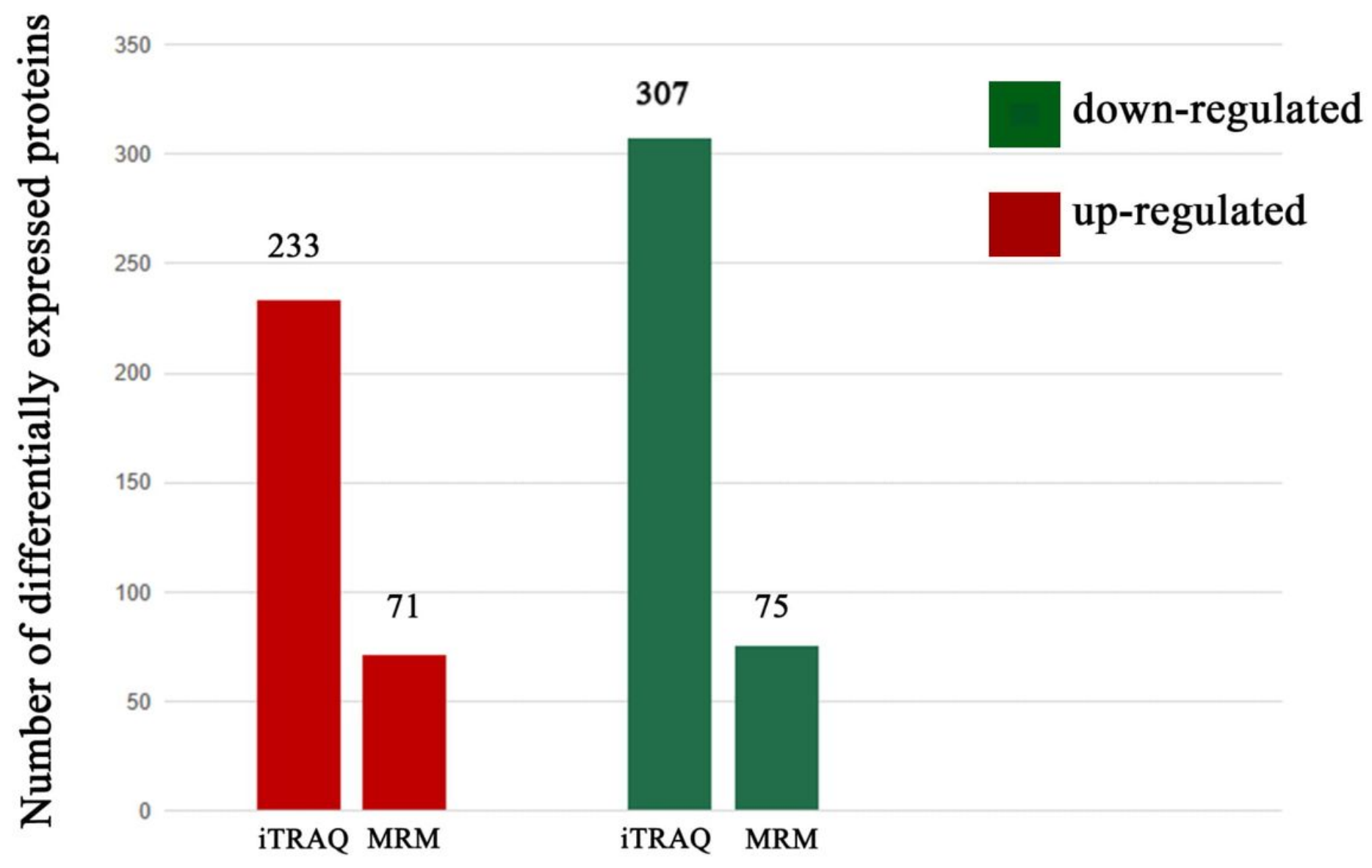

Figure 2

Statistics of the number of up and downregulated proteins identified by iTRAQ and MRM between 'FT' and cdm leaves 

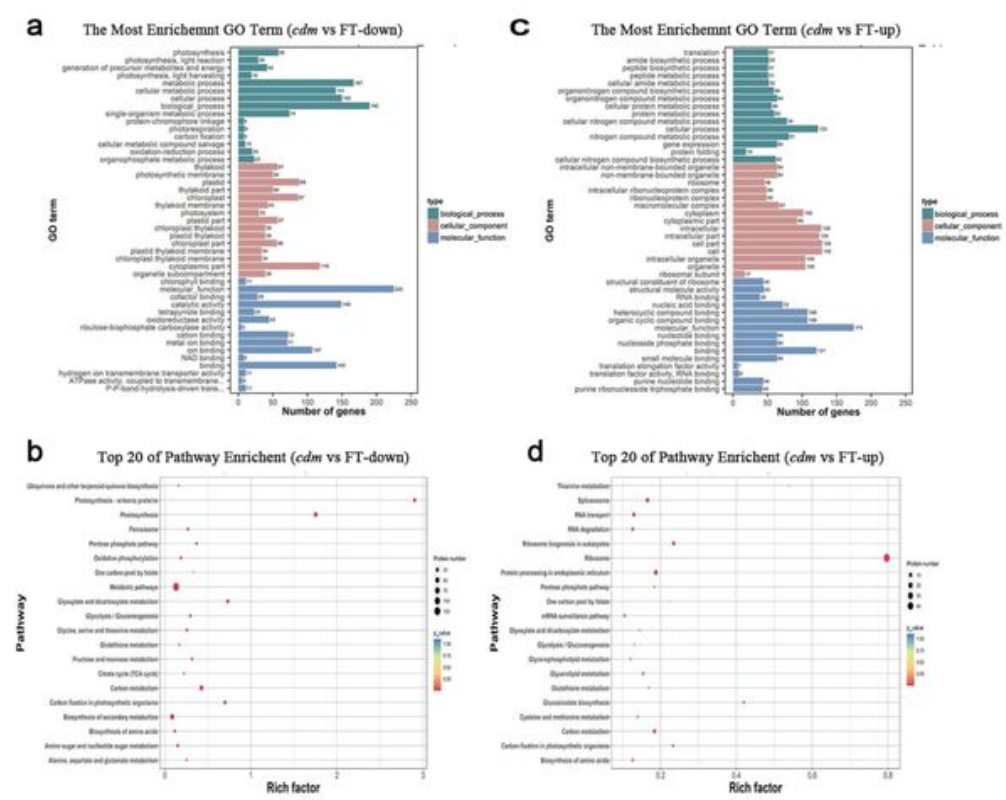

\section{Figure 3}

GO classification and KEGG pathway assignment of DRPs and URPs in 'FT' and cdm leaves. (a) GO classifications of DRPs, (b) KEGG pathway assignments of DRPs, (c) GO classifications of URPs, (d) KEGG pathway assignments of URPs 


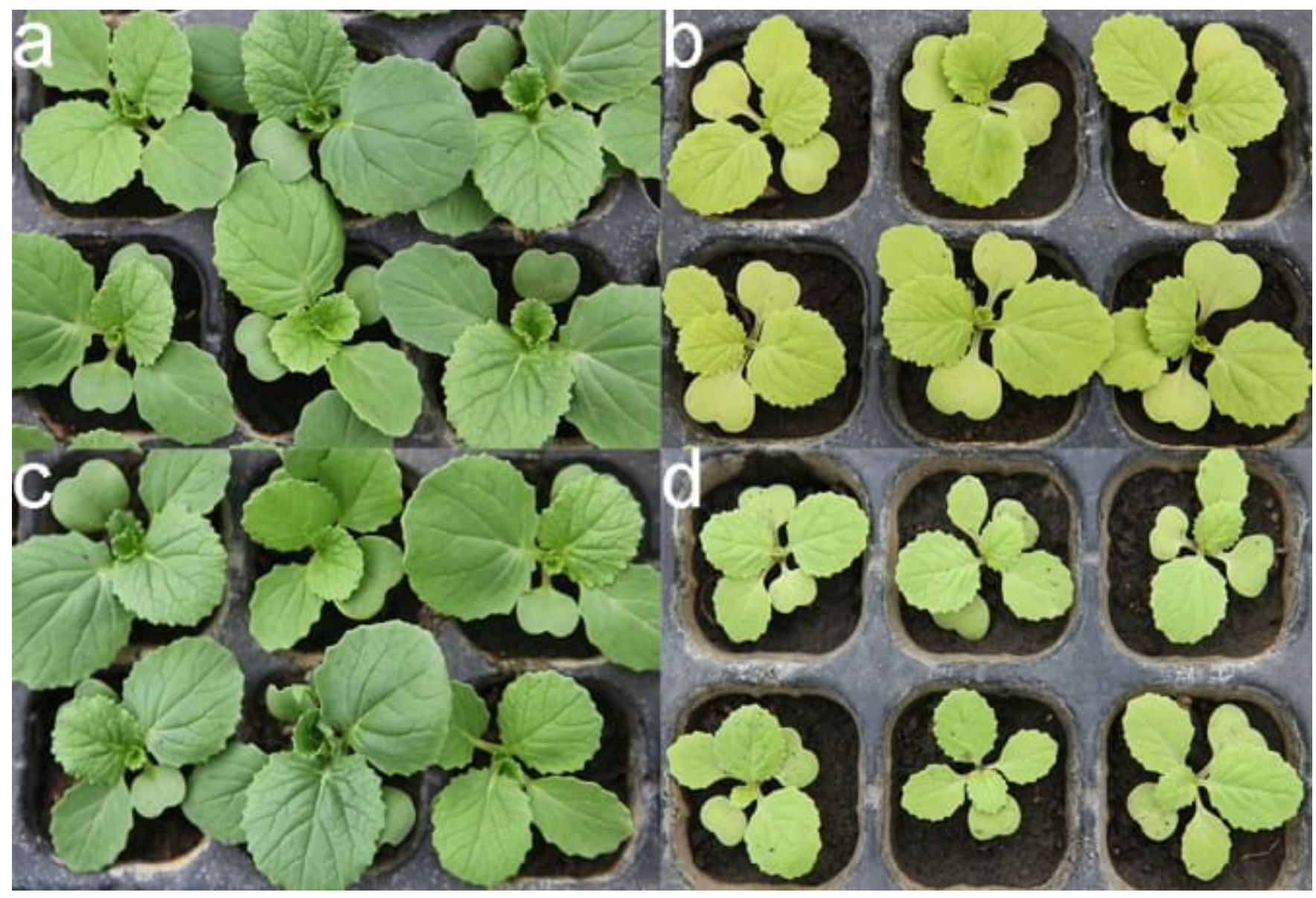

Figure 4

Comparison between ' $\mathrm{FT}$ ' and cdm plants grown at (a and b) $4^{\circ} \mathrm{C}$ or ( $\mathrm{c}$ and d) $26^{\circ} \mathrm{C}$ 
Bra000837

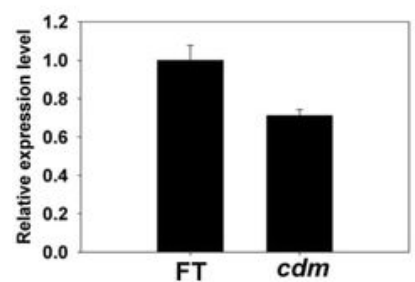

Bra012203

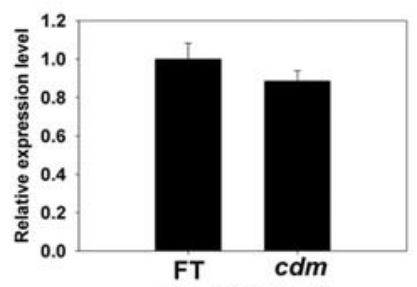

Bra015696

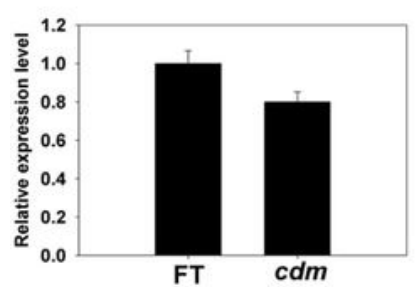

Bra032672

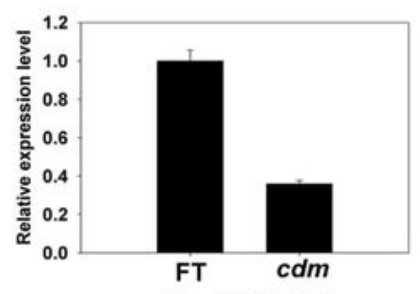

Bra038418

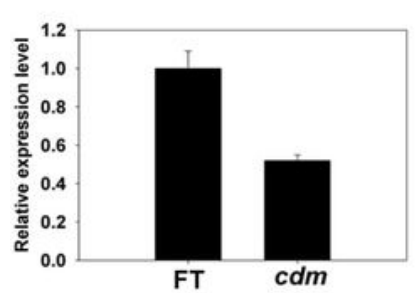

Bra010774

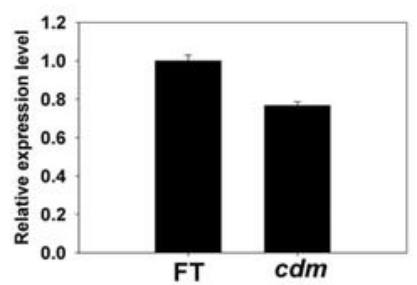

FT
Bra008392
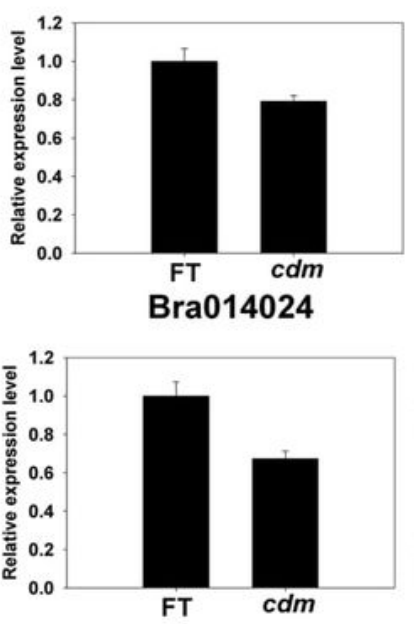

Bra019675
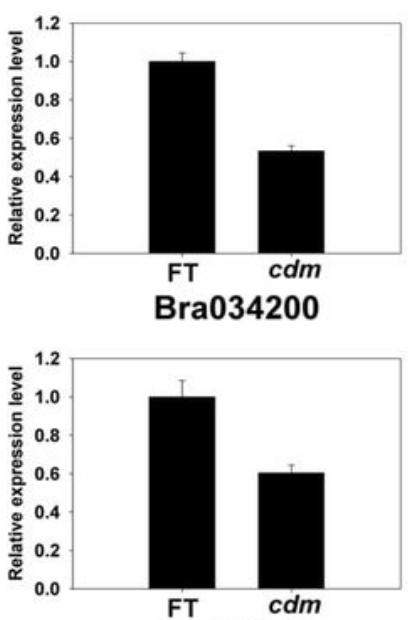

Bra036240

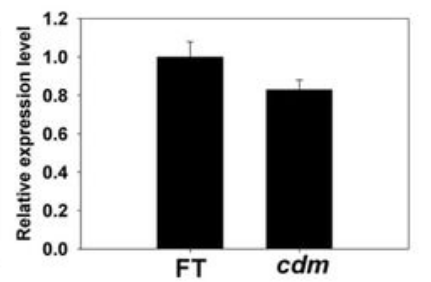

Bra015520

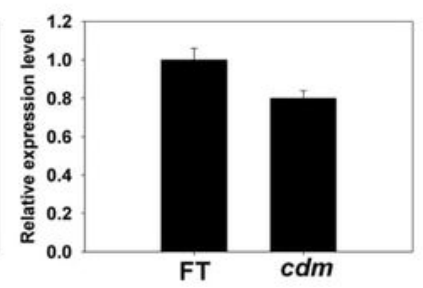

Bra010350
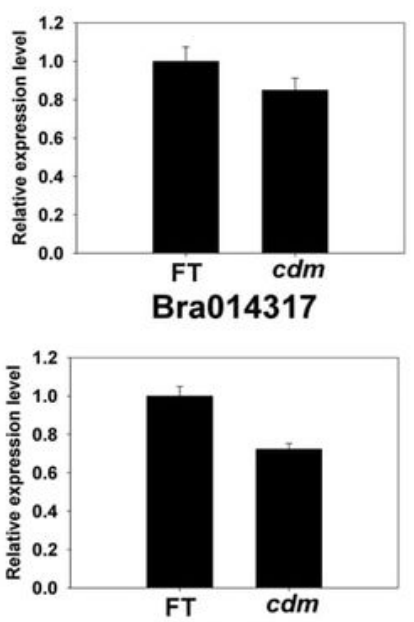

Bra029563

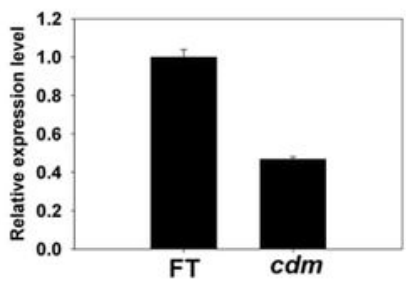

Bra037164
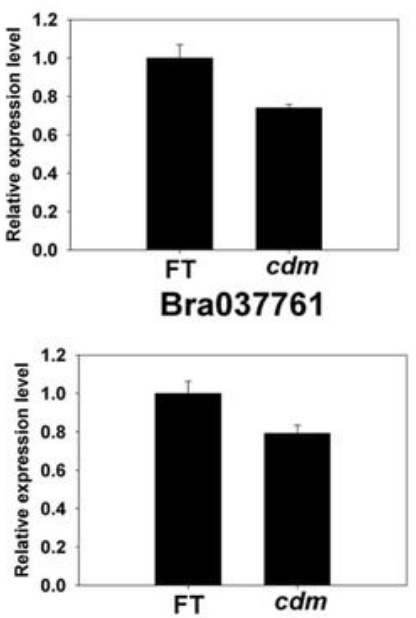

Bra030843

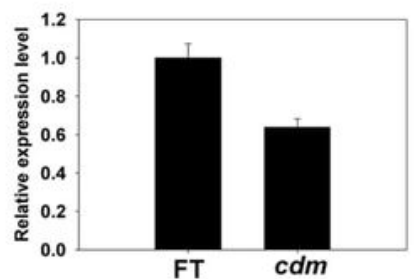

Figure 5

qRT-PCR of 18 DEPs in 'FT' and cdm leaves 


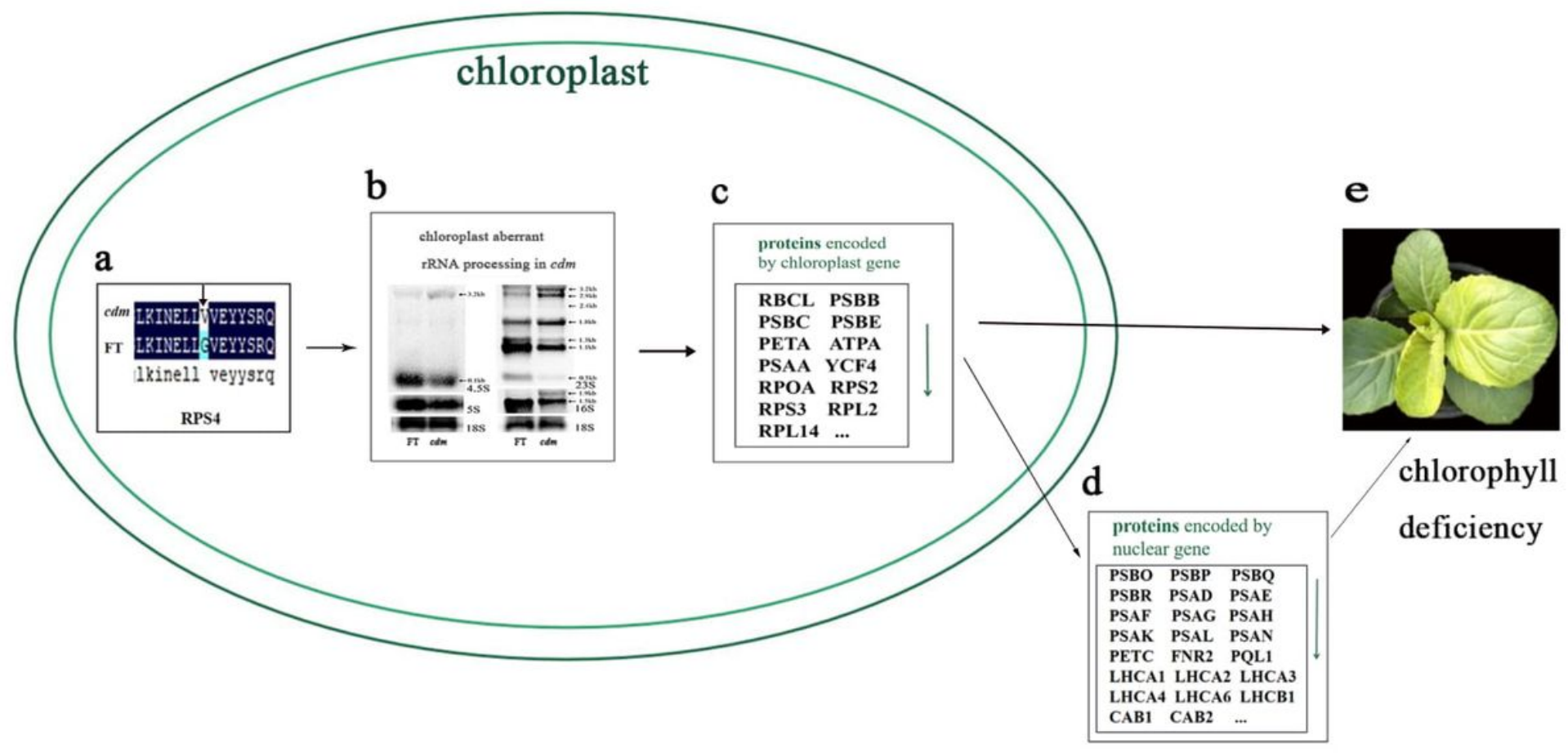

Figure 6

A hypothetical model for the chlorophyll deficiency mechanism in cdm. (a) The $G$ substitution for $V$ at residue 193 of chloroplast RPS4 in cdm, (b) Aberrant rRNA processing in cdm [31], (c) A number of proteins encoded by chloroplast genes are downregulated in cdm leaves, (d) A number of proteins encoded by nuclear genes are downregulated in cdm leaves, (e) cdm plants

\section{Supplementary Files}

This is a list of supplementary files associated with this preprint. Click to download.

- TableS11.xls

- Tables10.xls

- Tables9.xls

- Tables8.xls

- Tables7.xls

- Tables6.xls

- Tables5.xls

- Tables4.xls

- Tables3.xls

- Tables2.xls

- Tables1.xls 
- FigureS3.pdf

- Figures2.pdf

- FigureS1.pdf 